IUCrJ

ISSN 2052-2525

BIOLOGY|MEDICINE

Received 16 November 2021

Accepted 18 January 2022

Edited by K. Moffat, University of Chicago, USA

We dedicate this article to the Protein Data Bank on the occasion of the 50th anniversary of its commencement. Our article provides a detailed overview for the particular group of crystal structures in the PDB that involve rhenium compounds and also illustrates the wide-ranging impact that the PDB archive has, even with this one group, upon medical therapy and medical imaging.

Keywords: organometallic complexes; proteins; rhenium; technetium; radiopharmaceuticals; radioisotopes; transition metals.

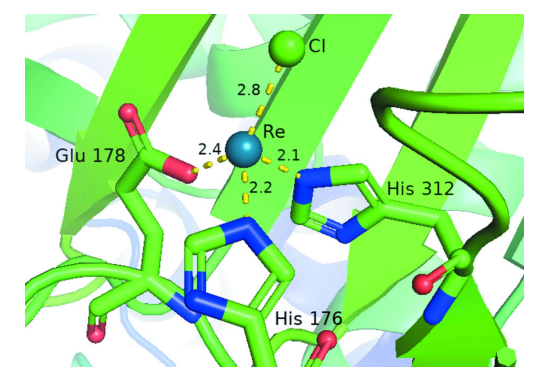
(c) () OP OPEN 2 ACCESS Published under a CC BY 4.0 licence

\section{Trends in coordination of rhenium organometallic complexes in the Protein Data Bank}

\author{
Alice Brink, $^{\mathrm{a}, \mathrm{b} *}$ Francois J. F. Jacobs ${ }^{\mathrm{a}}$ and John R. Helliwell ${ }^{\mathrm{b}}$ \\ ${ }^{a}$ Chemistry Department, University of the Free State, Nelson Mandela Drive, Bloemfontein, South Africa, and \\ ${ }^{\mathbf{b}}$ Department of Chemistry, The University of Manchester, Oxford Road, Manchester, United Kingdom. *Correspondence \\ e-mail: brinka@ufs.ac.za
}

Radiopharmaceutical development has similar overall characteristics to any biomedical drug development requiring a compound's stability, aqueous solubility and selectivity to a specific disease site. However, organometallic complexes containing ${ }^{188 / 186} \mathrm{Re}$ or ${ }^{99 \mathrm{~m}} \mathrm{Tc}$ involve a $d$-block transition-metal radioactive isotope and therefore bring additional factors such as metal oxidation states, isotope purity and half life into play. This topical review is focused on the development of radiopharmaceuticals containing the radioisotopes of rhenium and technetium and, therefore, on the occurrence of these organometallic complexes in protein structures in the Worldwide Protein Data Bank (wwPDB). The purpose of incorporating the group 7 transition metals of rhenium/technetium in the protein and the reasons for study by protein crystallography are described, as certain PDB studies were not aimed at drug development. Technetium is used as a medical diagnostic agent and involves the ${ }^{99 \mathrm{~m}} \mathrm{Tc}$ isotope which decays to release gamma radiation, thereby employed for its use in gamma imaging. Due to the periodic relationship among group 7 transition metals, the coordination chemistry of rhenium is similar (but not identical) to that of technetium. The types of reactions the potential model radiopharmaceutical would prefer to partake in, and by extension knowing which proteins and biomolecules the compound would react with in vivo, are needed. Crystallography studies, both small molecule and macromolecular, are a key aspect in understanding chemical coordination. Analyses of bonding modes, coordination to particular residues and crystallization conditions are presented. In our Forward look as a concluding summary of this topical review, the question we ask is: what is the best way for this field to progress?

\section{Introduction}

Drug development is a complex study involving multiple factors. The development of one new medical product, from its discovery to the time it is made available for the treatment of patients, takes on average 10-15 years, with an average cost of $\$ 800$ million to $\$ 1.6$ billion to research and develop each successful drug. For every drug that receives approval, an estimated 10000 compounds have entered the research and development pipeline and been discarded for particular reasons (PhRMA, 2015; Eckstein, 2005; Tonkens, 2005; Torjesen, 2015). Research typically selects a 'target' for a potential medicine, which is generally a single molecule (e.g. a gene or protein) that is involved in a particular disease. It is important to confirm how a chosen target is involved in the disease and whether it can interact and be affected by a drug molecule. The search for a promising molecule or 'lead compound' can now be undertaken with the hope that the disease can be arrested. Lead compounds are assessed early on for safety according to their pharmokinetics or absorption, 
distribution, metabolism, excretion and toxicity (ADME/Tox) properties, whereby the function and performance of hundreds of different compounds are tested in vitro (RuizGarcia et al., 2008; Tuntland et al., 2014; Chung et al., 2015). Due to the existence of libraries of compounds with known pharmacokinetic properties, it is possible to generate predictive models through machine-learning techniques. This has been successfully employed in pharmacokinetic studies and is helping the complex process of designing new drug candidates from the use of reliable machine-learning models and from studies of quantitative structure-activity relationships (Maltarollo et al., 2015).

Traditionally, medicinal chemistry has focused on organic, not inorganic, chemistry. The potential role of organometallic complexes, relatively speaking, has been neglected. The inclusion of metal atoms significantly increases the variety of building blocks which can be made, but at the same time increases the complexity of mechanistic behaviour, protein coordination, stability etc., hence the reason for their minority within the drug market (Nogrady \& Weaver, 2005). In radiopharmaceutical development, whereby the model complexes contain a radioactive isotope, additional factors must be considered such as isotope purity, half life, the cost and availability of the isotope as well as radiation dose (Liu, 2004). Our interest is in the development of radiopharmaceuticals containing the radioisotopes of technetium and rhenium. Technetium in the form of ${ }^{99 \mathrm{~m}} \mathrm{Tc}$ decays to release gamma radiation and is thereby employed for its use in gamma or single photon emission computed tomography (SPECT) imaging. It is widely utilized for diagnostic nuclear medicine with $80 \%$ of current radiopharmaceuticals administered clinically containing this radioisotope (Liu, 2004; Kluba \& Mindt, 2013). The ${ }^{99 \mathrm{~m}} \mathrm{Tc}$ isotope is routinely used in brain (Jurisson et al., 1993; Dilworth \& Parrott, 1998), heart (Holman et al., 1984; Gerson et al., 1983), bone (Dilworth \& Parrott, 1998) and thyroid (Dodds \& Powell, 1968) imaging. Moreover, the isotope is being investigated for selective cancer imaging and multidrug resistance (Piwnica-Worms et al., 1995; Herman et al., 1995; Goffin et al., 2017; Monteiro et al., 2017; Lin et al., 2018). Due to the chemical periodic relationship among group 7 transition metals, the coordination chemistry of rhenium is similar (but not identical) to that of technetium. This similarity is advantageous as it allows bifunctional chelating ligands that have been developed for ${ }^{99 \mathrm{~m}} \mathrm{Tc}$ to be used for rhenium and vice versa. The advantage of working first with rhenium is that experimental synthesis can be conducted on a non-radioactive 'cold' isotope (whereas all technetium isotopes are radioactive), and thus permits detailed chemical and reactivity analysis without the hazards of using the radioactive isotopes ${ }^{186 / 188} \mathrm{Re},{ }^{99 \mathrm{~m}} \mathrm{Tc}$ and ${ }^{99} \mathrm{Tc}$. There are two rhenium radionuclides utilized in therapeutic nuclear medicine, namely ${ }^{186} \mathrm{Re}$ and ${ }^{188} \mathrm{Re}$, which function by means of $\beta$-irradiation. Rhenium complexes have been developed for bone metastasis (Lam et al., 2009; Liepe et al., 2005), liver cancer (Lambert et al., 2005) and as steroid mimics (Chi \& Katzenellenbogen, 1993; Chi et al., 1994; DiZio et al., 1992). Investigating the fundamental chemical behaviour of a given chemical complex is necessary to predict what types of reactions the potential model radiopharmaceutical would partake in and, by extension, know which amino acids in proteins and biomolecules the compound would react with in vivo. These structure-function-reactivity studies, both within small-molecule and macromolecular research fields, are a key aspect to predicting and/or optimizing chemical coordination. These properties are utilized in the field of fragment-based drug development and high-throughput screening (Erlanson, 2012; Murray et al., 2012; Joseph-McCarthy et al., 2014).

The various differences between small-molecule and macromolecular crystallography methods, software and results often inhibit cross utilization, i.e. the interoperability of the data (Brink \& Helliwell, 2019b). New software-usage trends, such as with GOLD (Jones et al., 1997) or CSD-CrossMiner, both developed by the Cambridge Crystallographic Data Centre (CCDC) (Groom et al., 2016), are improving this interdisciplinary and interoperability data usage. This review focuses on protein structures found in the Protein Data Bank (PDB) that contain a rhenium or technetium metal centre. Our aims are to better understand:

(a) the chemical basis of this transition-metal family in its interactions with biological molecules;

(b) the effects that a non-natural metal may have within an organic macromolecular model;

(c) whether any possible chemical trends can be identified from the biological structural data; and

(d) the likely stability or even strict relevance of the measured structural data, such as that involving the specific crystallization conditions used and tabulated in Table 1. As a simple question we ask: does the $\mathrm{pH}$ used for crystallization match the $\mathrm{pH}$ values seen in the human body? For example, stomach acid $\mathrm{pH}$ is $\sim 1$ and the $\mathrm{pH}$ of the blood is 7.4. Many protein crystals are grown according to the general principles of macromolecular crystallization and crystal perfection, optimized by technique and technologies to measure the best possible diffraction data as described in Chayen et al. (2010). As Table 1 documents, a good fraction of the crystals are grown at or around $\mathrm{pH}$ 7. There are none for $\mathrm{pH} 1$ however, i.e. the relevant $\mathrm{pH}$ if a compound was to be administered orally and therefore should be chemically stable within the stomach.

In our Forward look as a concluding summary of this review, the question we ask is: what is the best way for this field to progress? Several chemical mechanistic studies have focused on protein-metal coordination and selectivity for rhenium (Zobi \& Spingler, 2012; Santoro et al., 2012; Takematsu et al., 2013; Brink \& Helliwell, 2019a, 2017; Binkley et al., 2011), for platinum (Messori \& Merlino, 2016; Tanley et al., 2014; Wang et al., 2017) or for rhodium (Loreto et al., 2021; Abe et al., 2009; Daubit et al., 2020). However, protein-ligand (i.e. the organic ligand bound to the organometallic complex) interactions may too play a role. Each of these mechanisms will have a direct effect on the viability of the complex as a radiopharmaceutical and on the design of the next iteration of potential model radiopharmaceuticals. Theoretical calculations describing the reactivity binding of rhenium complexes 
Table 1

Comparison of rhenium complexes bound to proteins as deposited in the PDB.

The PDB entries are listed relative to their appearance in the main text and therefore subdivided according to their application, namely (i) MAD or SAD phasing using relatively simple rhenium compounds, (ii) electron transfer and/or electron tunnelling and (iii) for medical applications.

(i) MAD or SAD phasing

\begin{tabular}{|c|c|c|c|c|c|}
\hline PDB entry & 3lya & $6 f 9 p$ & $1 \mathrm{hnu}$ & $1 \mathrm{k} 4 \mathrm{j}$ & $5 \mathrm{k} 1 \mathrm{j}$ \\
\hline Author & Eichinger et al. (2011) & Bastard et al. (2018) & Mursula et al. (2001) & Watson et al. (2002) & Ciccone et al. (2016) \\
\hline Rhenium complex & $\mathrm{K}_{2} \mathrm{ReCl}_{6}$ & $\mathrm{~K}_{2} \mathrm{ReCl}_{6}$ & Potassium perrhenate & Ammonium perrhenate & $\begin{array}{l}\text { Rhenium tris-carbonyl } \\
\text { pyta-C } 8 \text { derivative }\end{array}$ \\
\hline Method & $\mathrm{X}$-ray diffraction & X-ray diffraction & $\mathrm{X}$-ray diffraction & $\mathrm{X}$-ray diffraction & X-ray diffraction \\
\hline $\begin{array}{l}\text { Protein } \\
\text { macromolecule }\end{array}$ & $\begin{array}{l}\text { Transcriptional activator } \\
\text { CadC }\end{array}$ & L-Lysine 4-hydroxylase & $\begin{array}{l}\text { D3,D2-enoyl CoA isomerase } \\
\text { ECI1 }\end{array}$ & $\begin{array}{l}\text { Acyl-homoserinelactone } \\
\text { synthase (EsaI) }\end{array}$ & Human transthyretin \\
\hline Protein:Re ratio & $\mathrm{NS} \dagger$ & NS & $\begin{array}{l}1 \mathrm{~m} M \text { potassium perrhenate } \\
\left(\mathrm{KReO}_{4}\right)\end{array}$ & NS & NS \\
\hline $\begin{array}{l}\text { Crystallization } \\
\text { conditions }\end{array}$ & $\begin{array}{l}\text { Vapour-diffusion hanging } \\
\text { drop. CadC } \\
\text { ferred from mother liquor to } \\
\text { ferreds trans- } \\
2.5 M \mathrm{Li}_{2} \mathrm{SO}_{4} \text { to avoid the } \\
\text { formation of metal-ammonia } \\
\text { complexes. } 30 \mathrm{~m} M \mathrm{~K}_{2} \mathrm{ReCl}_{6} \\
\text { and } 1.7 M \mathrm{Li}_{2} \mathrm{SO}_{4} \text { cautiously } \\
\text { added to the drop until final } \\
\mathrm{ReCl}_{6}{ }^{2-} \text { concentration } \\
\text { reached } 5 \mathrm{mM}\end{array}$ & $\begin{array}{l}\text { 24\% PEG } 3350,0.2 \mathrm{M}_{\text {imida- }} \\
\text { zole malate, } 0.15 \mathrm{Mi}_{2} \mathrm{SO}_{4} \text {, } \\
\text { pH } 7.0\end{array}$ & $\begin{array}{l}\text { Vapour-diffusion hanging } \\
\text { drop in } 0.1 M \text { MES (pH 5.6), } \\
5 \%(w / v) 1,4 \text {-dioxane and } \\
1.4 M \text { ammonium sulfate }\end{array}$ & $\begin{array}{l}\text { Vapour-diffusion hanging- } \\
\text { drop methods with PEG } 4000 \text {, } \\
\text { 2-propanol, MES, EDTA, } \\
\beta \text {-mercaptoethanol, } \mathrm{NaN}_{3} \text {, } \\
\text { pH } 6.1\end{array}$ & $\begin{array}{l}21 \% \text { PEG } 4000 \text { (PEG } 4 \mathrm{~K} \text { ) } \\
\text { and } 0.14 M \text { imidazole malate, } \\
\text { pH } 6.0\end{array}$ \\
\hline Crystallization time & NS & NS & NS & NS & A few hours \\
\hline $\begin{array}{l}\text { Crystallization } \\
\text { temperature }\left({ }^{\circ} \mathrm{C}\right)\end{array}$ & 20 & 20 & 22 & 18 & 20 \\
\hline Cryoprotectant & $\begin{array}{l}2.5 \mathrm{M} \mathrm{Li}_{2} \mathrm{SO}_{4}, 30 \%(v / v) \\
\text { glycerol }\end{array}$ & $\begin{array}{l}30 \% \mathrm{CM} 5,26 \% \text { PEG } 3350, \\
0.2 \mathrm{M} \text { imidazole malate, pH } 8 \\
0.15 \mathrm{M} \mathrm{Li}_{2} \mathrm{SO}_{4}, 0.2 \mathrm{~m} M \\
\mathrm{ReK}_{2} \mathrm{Cl}_{6}, 20 \text { min soak }\end{array}$ & $20 \%(v / v)$ ethylene glycol & NS & $\begin{array}{l}\text { Soaking for } 10 \mathrm{~min} \text { in cryo- } \\
\text { protectant [ } 40 \% \text { SM2 }(12.5 \% \\
\text { ethylene glycol, } 12.5 \% \\
\text { glycerol, } 12.5 \% 1,2 \text {-propane- } \\
\text { diol, } 25 \% \text { DMSO and } 37.5 \% \\
\text { 1,4-dioxane) } 25 \% \text { PEG } 8 \mathrm{~K}] \\
\text { and } 0.2 \mathrm{~m} M \text { of the rhenium } \\
\text { complex }\end{array}$ \\
\hline $\begin{array}{l}\text { No. of X-ray } \\
\text { wavelengths } \\
\text { and values }(\AA)\end{array}$ & $\begin{array}{l}3(1.17652,1.17705 \\
\text { and } 1.07813)\end{array}$ & $1(1.1761)$ & $\begin{array}{l}4(1.1765,1.1773,1.1697 \\
\text { and } 1.1836)\end{array}$ & $\begin{array}{l}4(1.14,1.1724,1.1719 \\
\text { and } 1.19)\end{array}$ & $1(1.175919)$ \\
\hline Space group & $P 6_{1} 22$ & $P 2_{1} 2_{1} 2_{1}$ & $P 6_{3} 22$ & $\mathrm{P}_{3}$ & $P 2_{1} 2_{1} 2$ \\
\hline Resolution $(\AA)$ & 2.3 & 2.4 & 2.15 & 2.5 & 1.69 \\
\hline $\begin{array}{l}\text { Protein-metal } \\
\text { bonding } \ddagger\end{array}$ & - & Glu178, His176 and His312 & - & - & His88 \\
\hline $\begin{array}{l}\text { Protein-metal } \\
\text { complex weak } \\
\text { interactions } \S\end{array}$ & $\begin{array}{l}\text { His } 344, \text { Glu447, Thr229, } \\
\text { Asp225 and Trp450 }\end{array}$ & - & Asn239 and Leu126 & $\begin{array}{l}\text { Gln120, Lys161, Phe164, } \\
\text { Cys85, Ser143, Ser119, Ser71, } \\
\text { Tyr135 and Thr96 }\end{array}$ & - \\
\hline Notes & - & $\begin{array}{l}\text { An unidentified blob with } \\
\text { electron density less than } 5 \sigma \text { is } \\
\text { found at the rhenium metal } \\
\text { site at what appears to be an } \\
\text { open coordination position }\end{array}$ & $\begin{array}{l}\text { Crystals were soaked for } 4 \mathrm{~h} \\
\text { with } 1 \mathrm{mM} \mathrm{KReO}_{4}\end{array}$ & No MTZ files available & $\begin{array}{l}\text { Additional protein-rhenium } \\
\text { interactions are possibly } \\
\text { present (dependent on reso- } \\
\text { lution) but have not been } \\
\text { reported in the article. The } \\
\text { Re atom sits in a pocket } \\
\text { surrounded by Trp79, Thr } 75 \\
\text { and His } 90\end{array}$ \\
\hline
\end{tabular}

(ii) Electron transfer and/or electron tunnelling

\begin{tabular}{|c|c|c|c|c|}
\hline PDB entry & $6 \mathrm{mjs}$ & $6 \mathrm{mjt}$ & $6 \mathrm{mjr}$ & $2 \mathrm{i} 7 \mathrm{o}$ \\
\hline Author & Takematsu et al. (2019) & Takematsu et al. (2019) & Takematsu et al. (2019) & Shih et al. (2008) \\
\hline Rhenium complex $\dagger$ & $f a c-\left[\operatorname{Re}(\mathrm{CO})_{3}(\mathrm{dmp})\right]^{+}$ & $f a c-\left[\operatorname{Re}(\mathrm{CO})_{3}(\mathrm{dmp})\right]^{+}$ & $f a c-\left[\operatorname{Re}(\mathrm{CO})_{3}(\mathrm{dmp})\right]^{+}$ & $f a c-\left[\operatorname{Re}(\mathrm{CO})_{3}(\mathrm{dmp})\right]^{+}$ \\
\hline Method & X-ray diffraction & X-ray diffraction & X-ray diffraction & X-ray diffraction \\
\hline Protein:Re ratio & NS & NS & NS & NS \\
\hline \multirow[t]{2}{*}{ Crystallization conditions } & $\begin{array}{l}\text { Vapour-diffusion method. } \\
0.1 \mathrm{MNaOAc}, 120 \mathrm{~m} M /\end{array}$ & $\begin{array}{l}\text { Vapour-diffusion method. } \\
0.1 \mathrm{M} \mathrm{NaOAc}, 120 \mathrm{~m} M /\end{array}$ & $\begin{array}{l}\text { Vapour-diffusion method. } \\
0.1 M \mathrm{NaOAc}, 120 \mathrm{~m} M /\end{array}$ & $\begin{array}{l}\text { Vapour-diffusion hanging- } \\
\text { drop method. } 20-24 \% \text { PEG }\end{array}$ \\
\hline & $150 \mathrm{mM} \mathrm{Li}_{2} \mathrm{SO}_{4}$ and $48.6 \%$ & $150 \mathrm{mM} \mathrm{Li}_{2} \mathrm{SO}_{4}$, and $48.6 \%$ & $150 \mathrm{mM} \mathrm{Li}_{2} \mathrm{SO}_{4}$, and $48.6 \%$ & $4000,100 \mathrm{mM} \mathrm{LiNO}_{3}, 100 \mathrm{~m} M$ \\
\hline Crystallization time & NS & NS & NS & NS \\
\hline $\begin{array}{l}\text { Crystallization } \\
\text { temperature }\left({ }^{\circ} \mathrm{C}\right)\end{array}$ & 21 & 21 & 21 & 24.85 \\
\hline Cryoprotectant & NS & NS & NS & NS \\
\hline $\begin{array}{l}\text { Number of X-ray wavelengths } \\
\text { and values }(\AA)\end{array}$ & $1(1.00)$ & $1(1.5406)$ & $1(1.5406)$ & $1(0.972)$ \\
\hline Space group & $C 222_{1}$ & $C 2$ & $P 2_{1} 2_{1} 2_{1}$ & I222 \\
\hline Resolution $(\AA)$ & 1.85 & 1.893 & 2.012 & 1.5 \\
\hline
\end{tabular}


topical reviews

Table 1 (continued)

\begin{tabular}{|c|c|c|c|c|}
\hline PDB entry & $6 \mathrm{mjs}$ & $6 \mathrm{mjt}$ & $6 \mathrm{mjr}$ & $2 \mathrm{i} 7 \mathrm{o}$ \\
\hline Protein-metal bonding $\ddagger$ & His126 & His126 & His126 & His124 \\
\hline $\begin{array}{l}\text { Protein-metal complex weak } \\
\text { interactions } \S\end{array}$ & $\begin{array}{l}\text { Gln12, Ala119, Leu120, } \\
\text { Gln107, Trp124 and Trp122 }\end{array}$ & Trp124 & $\begin{array}{l}\text { Ala119, Gln107, Phe124 and } \\
\text { Trp122 }\end{array}$ & Trp122 \\
\hline Notes & - & - & - & - \\
\hline
\end{tabular}

\begin{tabular}{|c|c|c|c|c|}
\hline PDB entry & $1 \mathrm{i} 53$ & 1jzi & $1 \mathrm{r} 1 \mathrm{c}$ & $2 \mathrm{fnw}$ \\
\hline $\begin{array}{l}\text { Author } \\
\text { Rhenium complex }\end{array}$ & $\begin{array}{l}\text { Di Bilio et al. }(2001) \\
{\left[\operatorname{Re}(\mathrm{CO})_{3}(\mathrm{~L})\left(\mathrm{H}_{2} \mathrm{O}\right)\right](\mathrm{Otf})(\mathrm{L}=} \\
\left.\text { phen or } 4,7-\mathrm{Me}_{2} \text { phen }\right)\end{array}$ & $\begin{array}{l}\text { Crane et al. }(2001) \\
{\left[\operatorname{Re}^{\mathrm{I}}(\mathrm{CO})_{3}(1,10-\text { phen- }\right.} \\
\text { anthroline })(\mathrm{His} 83)]\end{array}$ & $\begin{array}{l}\text { Miller et al. }(2003) \\
{\left[\operatorname{Re}^{\mathrm{I}}(\mathrm{CO})_{3}(1,10-\text { phen- }\right.} \\
\text { anthroline })(\text { His } 107)]\end{array}$ & $\begin{array}{l}\text { Blanco-Rodríguez et al. (2006) } \\
\text { fac- }\left[\operatorname{Re}^{\mathrm{I}}(\mathrm{CO})_{3}(1,10-\text {-phen- }\right. \\
\left.\text { anthroline })\left(\mathrm{H}_{2} \mathrm{O}\right)\right] \mathrm{CF}_{3} \mathrm{SO}_{3}\end{array}$ \\
\hline Method & X-ray diffraction & X-ray diffraction & X-ray diffraction & $\mathrm{X}$-ray diffraction \\
\hline Protein & Azurin & Azurin & Azurin & Azurin \\
\hline Protein:Re ratio & NS & NS & NS & NS \\
\hline Crystallization conditions & $\begin{array}{l}\text { Crystals from } 2 \mu \mathrm{l} \text { drops } \\
\text { containing } 26 \mathrm{mg} \mathrm{ml}^{-1} \\
{\left[\mathrm{Re}(\mathrm{CO})_{3}\left(4,7-\mathrm{Me}_{2} \text { phen }\right)(\mathrm{Hi}-\right.} \\
\mathrm{s} 107)]^{+} \mathrm{AzCu}_{2}^{+} \text {in } 25 \mathrm{~m} M \text { HEPES } \\
\text { pH } 7.5 \text {, equilibrated against a } \\
500 \mu \mathrm{l} \text { reservoir containing } 20 \% \\
\text { PEG } 8000 \text { and } 100 \mathrm{~m} M \text { in pH } 8.0\end{array}$ & 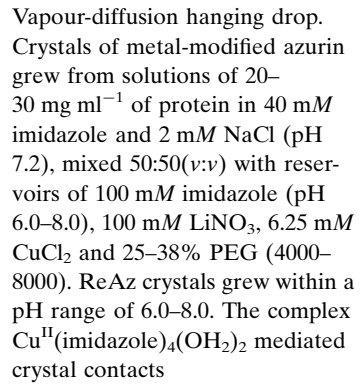 & $\begin{array}{l}\text { Vapour-diffusion sitting drop. } \\
\text { Crystals grew from } 2 \mu \text { drops } \\
\text { made from equal volumes of } \\
30 \mathrm{mg} \mathrm{ml}^{-1} \mathrm{ReAzCu}^{\mathrm{II}} \text { in } 25 \mathrm{~m} M \\
\mathrm{HEPES} \mathrm{pH} 7.5 \text { and reservoir. } \\
\text { Drops were treated with } 500 \mu \mathrm{l} \text { of } \\
\text { reservoir containing } 20 \% \text { PEG } \\
4000,100 \mathrm{~m} M \mathrm{LiNO}_{3} \text { and } 100 \mathrm{~m} M \\
\text { imidazole pH } 7.0\end{array}$ & $\begin{array}{l}\text { Vapour-diffusion hanging drop. } \\
\text { Crystals grew from } 2 \mu \mathrm{l} \text { drops } \\
\text { made from equal volumes of } \\
30 \mathrm{mg} \mathrm{ml}^{-1} \mathrm{Re}^{\mathrm{I}} \text {-azurin in } 25 \mathrm{~m} M \\
\text { HEPES pH } 7.5 \text { and reservoir. } \\
\text { Drops were treated with } 500 \mu \mathrm{l} \text { of } \\
\text { reservoir containing } 20 \% \mathrm{PEG} \\
4000,100 \mathrm{~m} M \mathrm{LiNO}_{3} \text { and } 100 \mathrm{mM} \\
\text { imidazole pH } 7.0\end{array}$ \\
\hline Crystallization time & NS & NS & NS & NS \\
\hline Crystallization temperature $\left({ }^{\circ} \mathrm{C}\right)$ & 21.85 & 20 & 25 & 25 \\
\hline Cryoprotectant & NS & NS & NS & NS \\
\hline $\begin{array}{l}\text { Number of X-ray wavelengths and } \\
\text { values }(\AA)\end{array}$ & $1(1.5418)$ & $1(1.08)$ & $1(0.9640)$ & $1(0.945)$ \\
\hline Space group & $P 1$ & $C 2$ & $P 2_{1}$ & $P 1$ \\
\hline Resolution $(\AA)$ & 1.80 & 1.62 & 1.9 & 1.4 \\
\hline Protein-metal bonding $\ddagger$ & His107 & His 83 and His 283 & His107 & $\begin{array}{l}\text { His } 83 \text { (renamed to His } 309 \text { ) and } \\
\text { His } 109\end{array}$ \\
\hline $\begin{array}{l}\text { Protein-metal complex weak } \\
\text { interactions } \S\end{array}$ & - & Val80 and Val280, & Ala53, His107 and Thr124 & $\begin{array}{l}\text { Lys122, Ala53, Lys322, Gln257 and } \\
\text { Ala253 }\end{array}$ \\
\hline Notes & No MTZ files available & No MTZ files available & No MTZ files available & No MTZ files available \\
\hline
\end{tabular}

\begin{tabular}{|c|c|c|c|}
\hline PDB entry & $4 \mathrm{k} 9 \mathrm{j}$ & 3ibo & $2 \mathrm{i} 7 \mathrm{~s}$ \\
\hline Author & Takematsu et al. (2013) & Blanco-Rodríguez et al. (2009) & Blanco-Rodríguez et al. (2009) \\
\hline Rhenium complex & $f a c-\left[\operatorname{Re}(\mathrm{CO})_{3}(\mathrm{dmp})\right]^{+}$ & $\begin{array}{l}\text { fac-[Re(imidazole })(\mathrm{CO})_{3}(1,10 \text {-phen- } \\
\text { anthroline })]_{2} \mathrm{SO}_{4} \cdot 4 \mathrm{H}_{2} \mathrm{O}\end{array}$ & $\begin{array}{l}\text { fac-[Re(imidazole })(\mathrm{CO})_{3}(1,10 \text {-phen- } \\
\text { anthroline })]_{2} \mathrm{SO}_{4} \cdot 4 \mathrm{H}_{2} \mathrm{O}\end{array}$ \\
\hline Method & X-ray diffraction & X-ray diffraction & $\mathrm{X}$-ray diffraction \\
\hline Protein macromolecule & Azurin & Azurin & Azurin \\
\hline Protein:Re ratio & $1: 1$ & NS & NS \\
\hline Crystallization conditions & $\begin{array}{l}\text { Vapour-diffusion sitting drop. Protein } \\
\text { buffer: } 40 \mathrm{~m} M \text { imidazole, } 2 \mathrm{~m} M \mathrm{NaCl} \text {. } \\
\text { Reservoir: } 100 \mathrm{~m} M \text { imidazole, } 100 \mathrm{~m} M \\
\text { LiNO }_{3}, 6.25 \mathrm{~m} M \mathrm{CuCl}_{2}, 27 \% \text { PEG } 4000 \text {, } \\
\text { pH } 7.4\end{array}$ & $\begin{array}{l}\text { Crystals grown from } 2.5 \mu \mathrm{l} \text { drops made } \\
\text { from } 1 \mu 130 \mathrm{mg} \mathrm{ml}^{-1} \mathrm{Re}^{\mathrm{I}} \text {-azurin in } 25 \mathrm{~m} M \\
\mathrm{HEPES} \mathrm{pH} 7.5,0.5 \mu \mathrm{l} \text { saturated } \\
{\left[\mathrm{Co}\left(\mathrm{NH}_{3}\right)_{5} \mathrm{Cl}\right] \mathrm{Cl}_{2} \text {, and } 1 \mu \mathrm{l} \text { of reservoir }} \\
\text { solution. Drops were equilibrated against } \\
500 \mu \mathrm{l} \text { reservoir containing } 20 \% \text { PEG } \\
4000,100 \mathrm{~m} M \mathrm{LiNO}_{3} \text { and } 100 \mathrm{~m} M \\
\text { imidazole pH } 7.0\end{array}$ & $\begin{array}{l}\text { Same conditions as 3ibo except that } \\
{\left[\mathrm{Co}\left(\mathrm{NH}_{3}\right)_{5} \mathrm{Cl}\right] \mathrm{Cl}_{2} \text { was not used }}\end{array}$ \\
\hline Crystallization time & $\begin{array}{l}\text { After six months, seed crystals were } \\
\text { harvested and used to grow larger crystals } \\
\text { over four months }\end{array}$ & NS & NS \\
\hline Crystallization temperature $\left({ }^{\circ} \mathrm{C}\right)$ & 24.85 & 24.85 & 24.85 \\
\hline Cryoprotectant & NS & NS & NS \\
\hline $\begin{array}{l}\text { Number of X-ray wavelengths and values } \\
(\AA)\end{array}$ & $1(0.9795)$ & $1(0.916)$ & $1(0.9480)$ \\
\hline Space group & $F 222$ & $P 12_{1} 1$ & $P 12_{1} 1$ \\
\hline Resolution $(\AA)$ & 1.7 & 1.45 & 1.35 \\
\hline Protein-metal bonding $\ddagger$ & His126 & His83, His109, His124 and His126 & His 124 , His 324 , His 524 and His724 \\
\hline $\begin{array}{l}\text { Protein-metal complex weak } \\
\text { interactions } \S\end{array}$ & - & Thr124, Gln107 and Thr21 & His124 \\
\hline Notes & - & - & - \\
\hline
\end{tabular}


Table 1 (continued)

(iii) Medical applications

\begin{tabular}{|c|c|c|c|c|}
\hline PDB entry & $1 b 0 q$ & $3 a x f$ & $3 r 26$ & $3 r j 7$ \\
\hline Author & Giblin et al. (1998) & Aryal et al. (2012) & Aryal et al. (2012) & Can et al. (2012) \\
\hline Rhenium complex & {$\left[\mathrm{ReOCl}_{3}\left(\mathrm{Me}_{2} \mathrm{~S}\right)\left(\mathrm{OPPh}_{3}\right)\right]$} & Sodium perrhenate & Sodium perrhenate & $\begin{array}{l}{\left[\left(\mathrm{CpCONH}_{-} \mathrm{CH}_{2} \mathrm{C}_{6} \mathrm{H}_{4-}-\right.\right.} \\
\left.\left.\mathrm{SO}_{2} \mathrm{NH}_{2}\right) \mathrm{Re}-(\mathrm{CO})_{3}\right]\end{array}$ \\
\hline Method & Solution NMR & X-ray diffraction & X-ray diffraction & X-ray diffraction \\
\hline Protein macromolecule & Cyclic $\alpha$-MSH & ModA & ModA & hCAII \\
\hline Protein:Re ratio & $1: 1$ & NS & NS & - \\
\hline Crystallization conditions & $\begin{array}{l}\text { 1:1 peptide-exchange complex } \\
\text { in } 62 \% \mathrm{MeOH}, \mathrm{pH} 8-9 \text { at } 65- \\
70^{\circ} \mathrm{C} \text { for } 1 \mathrm{~h}\end{array}$ & $\begin{array}{l}\text { Vapour-diffusion hanging } \\
\text { drop. } 26 \% \text { PEG } 8000,0.1 \mathrm{M} \\
\text { sodium acetate and } 2.5 \mathrm{mM} \\
\text { sodium perrhenate, pH } 5.0 \\
\text { solution }\end{array}$ & $\begin{array}{l}\text { Vapour-diffusion hanging } \\
\text { drop. } 26 \% \text { PEG } 8000,0.1 \mathrm{M} \\
\text { sodium acetate and } 2.5 \mathrm{mM} \\
\text { sodium perrhenate, pH } 5.0 \\
\text { solution }\end{array}$ & $\begin{array}{l}2.4 \mathrm{M}\left(\mathrm{NH}_{4}\right)_{2} \mathrm{SO}_{4}, 0.1 M \\
\text { Tris- } \mathrm{HCl} \text { at pH 7.5, } 1 \mathrm{~m} M 4- \\
\text { (hydroxymercuri) benzoic } \\
\text { acid sodium salt and } 5.1 \% \text { vol. } \\
\text { DMSO. hCAII crystals grown } \\
\text { with the hanging-drop vapour- } \\
\text { diffusion method and soaked } \\
\text { with the respective inhibitor }\end{array}$ \\
\hline Crystallization time & $\mathrm{NA} \dagger$ & Two weeks & Two weeks & $\begin{array}{l}\text { Four weeks at } 298 \mathrm{~K} \text {, or two } \\
\text { weeks at } 277 \mathrm{~K} \text { then again four } \\
\text { weeks at } 298 \mathrm{~K}\end{array}$ \\
\hline $\begin{array}{l}\text { Crystallization temperature } \\
\left({ }^{\circ} \mathrm{C}\right)\end{array}$ & NA & 25 & 25 & 25 or 4 \\
\hline Cryoprotectant & NA & $\begin{array}{l}10 \% \text { glycerol, } 27.5 \% \text { PEG } \\
8000 \text { and } 0.1 M \text { sodium acetate } \\
\text { (pH } 4.5 \text { ) for the wild-type } \\
\text { protein; and } 10 \% \text { glycerol, } \\
26 \% \text { PEG } 8000 \text { and } 0.1 M \\
\text { sodium acetate (pH } 5.0 \text { ) for } \\
\text { the mutant }\end{array}$ & $\begin{array}{l}10 \% \text { glycerol, } 27.5 \% \text { PEG } \\
8000 \text { and } 0.1 M \text { sodium acetate } \\
\text { (pH } 4.5 \text { ) for the wild-type } \\
\text { protein and } 10 \% \text { glycerol, } \\
26 \% \text { PEG } 8000 \text { and } 0.1 M \\
\text { sodium acetate (pH } 5.0 \text { ) for } \\
\text { the mutant }\end{array}$ & Paratone N \\
\hline $\begin{array}{l}\text { Number of X-ray wavelength } \\
\text { and values }(\AA)\end{array}$ & NA & $1(0.97857)$ & $1(0.97857)$ & $1(1.00)$ \\
\hline Space group & NA & $P 2_{1} 2_{1} 2_{1}$ & $P 322_{1}$ & $P 12_{1} 1$ \\
\hline Resolution $(\AA)$ & NA & 2.0 & 1.7 & 1.2 \\
\hline Protein-metal bonding $\ddagger$ & Cys1, Cys7, and Tryp6 & - & - & $\begin{array}{l}\mathrm{Zn} \text { atom found in the active } \\
\text { site. No formal protein- } \\
\text { rhenium bonds observed }\end{array}$ \\
\hline $\begin{array}{l}\text { Protein-metal complex weak } \\
\text { interactions§ }\end{array}$ & - & $\begin{array}{l}\text { Pro124, Ala125, Tyr170, Ser12, } \\
\text { Val152 and Ser39 }\end{array}$ & $\begin{array}{l}\text { Ser12, Ser39, Tyr170 and } \\
\text { Val152 }\end{array}$ & $\begin{array}{l}\text { Thr199, Leu198, Val121, } \\
\text { His15, Asp19, His4 and Asn11, } \\
\text { Phe131, Pro202 }\end{array}$ \\
\hline Notes & $\begin{array}{l}\text { In the PDB ligand view, the } \\
\text { nitrogen to rhenium inter- } \\
\text { actions are not indicated as the } \\
\text { data are sourced from NMR. } \\
\text { From a coordination-chem- } \\
\text { istry perspective, these inter- } \\
\text { actions should be reasonably } \\
\text { strong and present as they } \\
\text { complete the metal coordina- } \\
\text { tion sphere }\end{array}$ & - & - & $\begin{array}{l}\text { The deprotonated nitrogen of } \\
\text { the rhenium arylsulfonamide } \\
\text { complex terminus coordinates } \\
\text { to the } \mathrm{Zn} \text { atom }\end{array}$ \\
\hline
\end{tabular}

\begin{tabular}{|c|c|c|c|}
\hline PDB entry & $3 \mathrm{kam}$ & 3qng & $3 q \mathrm{qe} 8$ \\
\hline Author & Binkley et al. $(2010,2011)$ & Zobi \& Spingler (2012) & Zobi \& Spingler (2012) \\
\hline Rhenium complex & $f a c-\left[\operatorname{Re}(\mathrm{CO})_{3}\left(\mathrm{H}_{2} \mathrm{O}\right)_{3}\right] \mathrm{Br}$ & $\begin{array}{l}f a c-\left[\operatorname{Re}(\mathrm{CO})_{3}\left(\mathrm{H}_{2} \mathrm{O}\right)_{3}\right]^{+} \text {with } \mathrm{L}^{-} \\
\text {serine }\end{array}$ & $\begin{array}{l}\text { fac- }\left[\mathrm{Re}(\mathrm{CO})_{3}\left(\mathrm{H}_{2} \mathrm{O}\right)_{3}\right]^{+} \text {with } \\
\text { imidazole }\end{array}$ \\
\hline Method & X-ray diffraction & X-ray diffraction & $\mathrm{X}$-ray diffraction \\
\hline Protein macromolecule & $\begin{array}{l}\text { Hen egg-white lysozyme } \\
\text { (HEWL) }\end{array}$ & HEWL & HEWL \\
\hline Protein:Re ratio & $1: 5$ & $1: 10$ & $1: 10$ \\
\hline Crystallization conditions & $\begin{array}{l}0.05 M \text { MES buffer ( } \mathrm{pH} 5.5) \\
\text { and } 0.8 \mathrm{M} \mathrm{NaCl}\end{array}$ & $\begin{array}{l}0.05 \mathrm{M} \text { acetate buffer }(\mathrm{pH} 4.6) \\
\text { and } 0.9 \mathrm{M} \mathrm{NaCl}\end{array}$ & $\begin{array}{l}0.05 \mathrm{M} \text { acetate buffer }(\mathrm{pH} 4.6) \\
\text { and } 0.9 \mathrm{M} \mathrm{NaCl}\end{array}$ \\
\hline Crystallization time & $48-72 \mathrm{~h}$ & - & - \\
\hline $\begin{array}{l}\text { Crystallization temperature } \\
\left({ }^{\circ} \mathrm{C}\right)\end{array}$ & 19 & 25 & 25 \\
\hline Cryoprotectant & $30 \%$ glycerol & $25 \%$ glycerol & $25 \%$ glycerol \\
\hline $\begin{array}{l}\text { Number of X-ray wavelengths } \\
\text { and values }(\AA)\end{array}$ & $1(1.54)$ & $1(1.00)$ & $1(1.00)$ \\
\hline Space group & $P 4_{3} 22_{1}$ & $P 4_{3} 2_{1} 2$ & $P 2_{1} 2_{1} 2_{1}$ \\
\hline Resolution $(\AA)$ & 1.59 & 1.55 & 1.49 \\
\hline Protein-metal bonding $\ddagger$ & His 15 & His15 & His 15 \\
\hline $\begin{array}{l}\text { Protein-metal complex weak } \\
\text { interactions } \S\end{array}$ & - & Ile88, Asp87 and Thr89 & Ile88 \\
\hline Notes & $\begin{array}{l}\text { Soaking experiment yielded } \\
\text { identical results }\end{array}$ & - & - \\
\hline
\end{tabular}


Table 1 (continued)

\begin{tabular}{|c|c|c|c|}
\hline PDB entry & $5 \mathrm{nbj}$ & 6ro3 & 6ro5 \\
\hline Author & Brink \& Helliwell (2017) & Brink \& Helliwell (2019a) & Brink \& Helliwell (2019a) \\
\hline Rhenium complex & $f a c-\left[\operatorname{Re}(\mathrm{CO})_{3}\left(\mathrm{H}_{2} \mathrm{O}\right)_{3}\right]^{+}$ & $\begin{array}{l}f a c-\left[\operatorname{Re}(\mathrm{CO})_{3}\left(\mathrm{H}_{2} \mathrm{O}\right)_{3}\right]^{+} \text {and } \\
f a c-\left[\operatorname{Re}_{4}\left(\mu_{3}-\mathrm{OH}\right)_{4}(\mathrm{CO})_{12}\right]\end{array}$ & $\begin{array}{l}f a c-\left[\operatorname{Re}(\mathrm{CO})_{3}\left(\mathrm{H}_{2} \mathrm{O}\right)_{3}\right]^{+} \text {and } \\
f a c-\left[\operatorname{Re}_{4}\left(\mu_{3}-\mathrm{OH}\right)_{4}(\mathrm{CO})_{12}\right]\end{array}$ \\
\hline Method & X-ray diffraction & X-ray diffraction & X-ray diffraction \\
\hline Protein macromolecule & HEWL & HEWL & HEWL \\
\hline Protein:Re ratio & $1: 30$ & $1: 30$ & $1: 30$ \\
\hline Crystallization conditions & $\begin{array}{l}10 \% \mathrm{NaCl}, 0.04 M \text { sodium acetate }(\mathrm{pH} 4.7) \\
\text { and } f a c-\left[\mathrm{Et}_{4} \mathrm{~N}\right]_{2}\left[\mathrm{Re}(\mathrm{CO})_{3}(\mathrm{Br})_{3}\right] \text { at } 0.03 M \text { in } \\
1.4 \mathrm{ml} \text { water with DMSO [at } 7.5 \%(v / v)]\end{array}$ & $\begin{array}{l}10 \% \mathrm{NaCl}, 0.04 M \text { sodium acetate }(\mathrm{pH} 4.7) \\
\text { and } f a c-\left[\mathrm{Et}_{4} \mathrm{~N}\right]_{2}\left[\mathrm{Re}(\mathrm{CO})_{3}(\mathrm{Br})_{3}\right] \text { at } 0.03 \mathrm{M} \text { in } \\
1.4 \mathrm{ml} \text { water with DMSO [at } 7.5 \%(v / v)]\end{array}$ & $\begin{array}{l}10 \% \mathrm{NaCl}, 0.04 \mathrm{M} \text { sodium acetate }(\mathrm{pH} 4.7) \\
\text { and } f a c-\left[\mathrm{Et}_{4} \mathrm{~N}\right]_{2}\left[\operatorname{Re}(\mathrm{CO})_{3}(\mathrm{Br})_{3}\right] \text { at } 0.03 M \text { in } \\
1.4 \mathrm{ml} \text { water with DMSO }[\text { at } 7.5 \%(v / v)]\end{array}$ \\
\hline Crystallization time & Approximately three weeks & $\begin{array}{l}\text { Crystals were grown over approximately } \\
\text { three weeks and left undisturbed for two } \\
\text { years before collection }\end{array}$ & $\begin{array}{l}\text { Crystals were grown over approximately } \\
\text { three weeks and left undisturbed for a year } \\
\text { before collection }\end{array}$ \\
\hline Crystallization temperature $\left({ }^{\circ} \mathrm{C}\right)$ & 25 & 25 & 25 \\
\hline Cryoprotectant & Silicone oil & Silicone oil & Silicone oil \\
\hline $\begin{array}{l}\text { Number of X-ray wavelengths and values } \\
(\AA)\end{array}$ & $2(0.97625,1.5418)$ & $1(0.9763)$ & $1(0.9763)$ \\
\hline Space group & $P 4_{3} 2_{1} 2$ & $P 4_{3} 2{ }_{1} 2$ & $P 2_{1} 2_{1} 2_{1}$ \\
\hline Resolution $(\AA)$ & 1.266 & 1.03 & 1.68 \\
\hline Protein-metal bonding $\ddagger$ & $\begin{array}{l}\text { His15, Leu129, Asp119, Asp18, Glu35, } \\
\text { Asp52 }\end{array}$ & His15, Asp119 and Glu7 & $\begin{array}{l}\text { His15, Asp52, Glu35, Asp119, Asp18 and } \\
\text { Leu129 }\end{array}$ \\
\hline $\begin{array}{l}\text { Protein-metal complex weak } \\
\text { interactions } \S\end{array}$ & Arg125, Glu7 and Arg61 & Pro70, Arg5, Trp123, Lys33 and Asp87 & $\begin{array}{l}\text { Glu7, Lys1, Arg125, Asn46, Ile88, Thr89 and } \\
\text { Asp87, Leu129 }\end{array}$ \\
\hline Notes & - & - & $\begin{array}{l}f a c-\left[\mathrm{Re}_{4}\left(\mu_{3}-\mathrm{OH}\right)_{4}(\mathrm{CO})_{12}\right] \text { cluster is found as } \\
\text { a non-coordinating entity }\end{array}$ \\
\hline
\end{tabular}

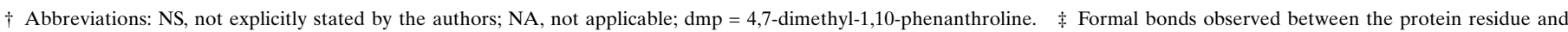
metal centre as indicated by the authors. $\S$ As viewed with RCSB PDB's NGL ligand viewer and mentioned by the authors.

to biomolecules (Oliveira et al., 2013; Aliyan et al., 2017; Carreño et al., 2021), as well as continued structural evaluations of future PDB entries, would indicate if there are new trends forming a mechanistic driving-force preferential for either protein-metal or protein-ligand coordination.

\section{Overview of the purposes of the depositors of these crystal structures}

Table 1 presents all the rhenium-bound protein crystal structures in the wwPDB including key crystallographic and synthetic aspects. A list of amino-acid residues directly bound to the rhenium metal centre as well as weak interactions from the protein to the organic ligand of the organometallic complex are specified. Where the structure factors were made available we have also examined any uninterpreted residualdifference electron-density features and offer appropriate comments. We also include the crystallization conditions used for each PDB entry.

The purposes of the protein studies listed in the wwPDB that contain rhenium metal centres show a variety of applications. These have included (i) multi- or single-wavelength anomalous dispersion (MAD or SAD) phasing using relatively simple rhenium compounds, (ii) electron transfer and/or electron tunnelling or (iii) for medical applications. We describe all three aspects and highlight key observations made either by the authors or through our examination of the data. Many studies have included the investigation of both rhenium and technetium with their respective biological activity and confirmed the presence of technetium via alternative methods of characterization (i.e. NMR, infra-red spectroscopy or mass spectrometry). However, the protein structures currently available in the PDB only include rhenium, not technetium.

\subsection{Rhenium for MAD or SAD phasing}

The purpose of rhenium in these protein structures was for MAD or SAD phasing and not the medical application of the metal's effects. However, that said, within this review's objective of extracting coordination data, these protein structures make a valuable contribution to possible trends for preferential binding sites and thereby expand the medicalapplication potential. The protein structures (with PDB codes and citations given in parentheses) that involved rhenium for phasing used relatively simple rhenium compounds: $\left[\mathrm{ReCl}_{6}\right]^{2-}$ (3lya, Eichinger et al., 2011; 6f9p, Bastard et al., 2018), perrhenate $\left[\mathrm{ReO}_{4}\right]^{-}(1 \mathrm{hnu}$, Mursula et al. $2001 ; 1 \mathrm{k} 4 \mathrm{j}$, Watson et al., 2002) or $f a c-\left[\operatorname{Re}(\mathrm{CO})_{3}\right]^{+}(5 \mathrm{k} 1 \mathrm{j}$, Ciccone et al., 2016), all of which are commercially available starting complexes and are readily soluble in water. Hence, interest in the specific chemistry of rhenium (i.e. oxidation states, coordination, stability etc.) was not considered a research priority of these particular studies. Variation in protein-metal coordination is found across this group, such as for $1 \mathrm{hnu}$ where rhenium is bound to the active site of the enoyl-CoA isomerase. Structure $5 \mathrm{k} 1 \mathrm{j}$ has rhenium coordinated at His88, as well as being present at multiple sites in varying low occupancy. The study 3lya altogether shows 16 bound $\left[\mathrm{ReCl}_{6}\right]^{2-}$ ions to residues including histidine, tryptophan, aspartic and glutamic acid. The study $6 \mathrm{f} 9 \mathrm{p}$, also utilizing $\left[\mathrm{ReCl}_{6}\right]^{2-}$, found that the rhenium retains only one chloride and its ligands are replaced by amino-acid interactions, notably two histidines, i.e. a special case for this particular protein (Fig. 1).

2.2. Rhenium-based crystal structures for studying electron transfer and/or tunnelling

Electron tunnelling is a quantum mechanical phenomenon that occurs when electrons move through a barrier that 
classically should not be possible to traverse. In proteins, electron tunnelling can move electrons between donor and acceptor sites separated by distances ranging from 10 to $30 \AA$ on a millisecond or even femtosecond time scale (Stuchebrukhov, 2010; Tezcan et al., 2001). As an example, protein structures 2i7o (Shih et al., 2008), 6mjs, 6mjt and 6mjr (Takematsu et al., 2019) reported the use of a rhenium(I) tricarbonyl complex, fac- $\left[\operatorname{Re}^{\mathrm{I}}(\mathrm{CO})_{3}\right]$, in a mutant Pseudomonas aeruginosa azurin to examine the electron-transfer capability between distant metal redox centres within the protein. In $2 \mathrm{i} 7 \mathrm{o}$ the rhenium complex $\left[\operatorname{Re}^{\mathrm{I}}(\mathrm{CO})_{3}(\mathrm{dmp})\right](\mathrm{dmp}=4,7-\mathrm{di}-$ methyl-1,10-phenanthroline) was attached to the histidine-124

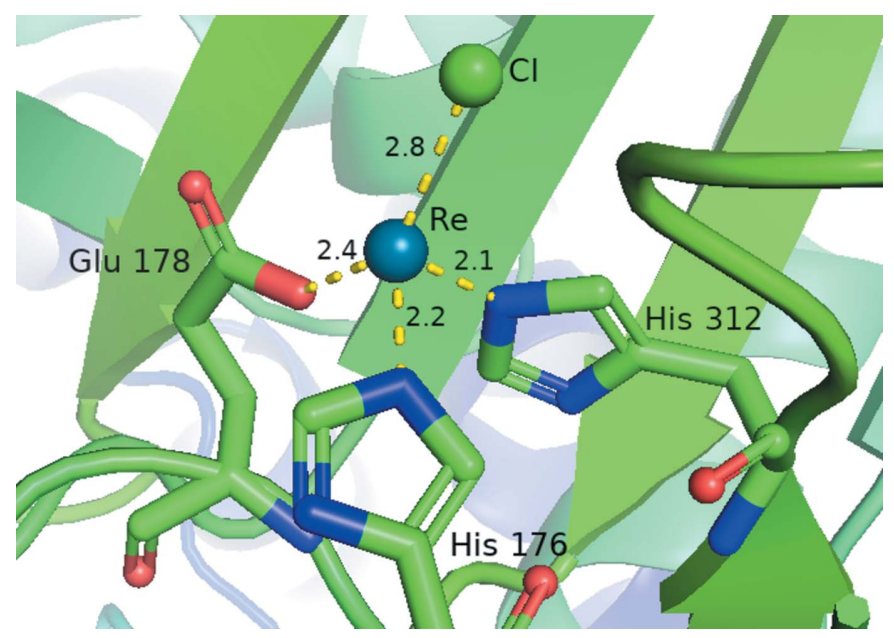

Figure 1

A coordination-site view of $6 f 9 p$ at one of the four rhenium sites. The other sites are equivalent to the one shown. This figure has been made using PyMOL (DeLano, 2002). In this and all figures carbon atoms are indicated as green (as are the ribbon diagrams for protein structures), nitrogens are blue, oxygens are red, sulfur is yellow, rheniums are cyan, copper is orange and chlorine is dark green. Hydrogen atoms are generally omitted due to the lower-resolution data typically obtained by macromolecular crystallographic investigations; however, in this topical review, when their positions are more accurately known, such as in the case of $1 \mathrm{~b} 0 \mathrm{q}$, hydrogen atoms are coloured light grey. The distances shown are in $\AA$. residue (Fig. 2). The $1.5 \AA$ resolution crystal structure of the Re-labelled protein shows that the ligand (dmp) and the trypophan-122 indole group are near van der Waals interaction distances $(\sim 4 \AA)$, and the $\mathrm{Cu}-\mathrm{Re}$ distance is $19.4 \AA$. Structures $6 \mathrm{mjs}$, $6 \mathrm{mjt}$ and $6 \mathrm{mjr}$ are coordinated to the His 126 via the imidazole $\mathrm{N}$ ring bonded to the octahedral fac$\left[\operatorname{Re}(\mathrm{CO})_{3}\right]^{+}$moiety in the sixth ligand position. Additional studies [1153, Di Bilio et al. (2001); 1jzi, Crane et al. (2001); 1r1c, Miller et al. (2003); 2fnw, Blanco-Rodríguez et al. (2006); 4k9j, Takematsu et al. (2013); 3ibo, Blanco-Rodríguez et al. (2006); 2i7s, Blanco-Rodríguez et al. (2009)] similarly show the direct coordination of the $f a c$ - $\left[\operatorname{Re}^{\mathrm{I}}(\mathrm{CO})_{3}\right]$ core to the protein via the histidine imidazole moiety.

\subsection{Medical applications}

Technetium and rhenium can exist in a range of oxidation states ranging from +7 to -1 (rhenium can range further to -3). Due to technetium's $\left({ }^{99 \mathrm{~m}} \mathrm{Tc}\right)$ ideal radiodiagnostic properties (i.e. a half life of $6.02 \mathrm{~h}$, gamma radiation of $141 \mathrm{keV}$ and sourced from a ${ }^{99} \mathrm{Mo}-{ }^{99 \mathrm{~m}} \mathrm{Tc}$ generator) (Firestone et al., 1996; Boswell \& Brechbiel, 2007), as well as rhenium's similarity in chemistry, including its own ${ }^{188 / 186} \mathrm{Re}$ isotope used for therapy, these elements have been extensively investigated for medical applications. Multiple generations of complexes have been developed utilizing various oxidation states and cores, such as pertechnetate, ${ }^{99 \mathrm{~m}} \mathrm{TcO}_{4}^{-}$(in the +7 oxidation state and commercially available as TechneLite) (Dodds \& Powell, 1968), and the $\mathrm{Tc}^{+5}$ mono-oxo $(\mathrm{Tc}=\mathrm{O})$ core (Ceretec) (Mazzi et al., 2007). The ${ }^{99 \mathrm{~m}} \mathrm{Tc}-\mathrm{MDP}$ also known as ${ }^{99 \mathrm{~m}} \mathrm{Tc}-$ medronate (Osteolite) used for imaging of bone metastasis has a +4 oxidation state and is thought to coordinate in an octahedral fashion. Furthermore, ${ }^{99 \mathrm{~m}} \mathrm{Tc}$-tetrofosmin (Myoview) (Kelly et al., 1993) is a cationic compound and is used in myocardial perfusion imaging. It contains a $\mathrm{Tc}^{+5}$ trans di-oxo $\left(\mathrm{Tc}=\mathrm{O}_{2}\right)$ core. Moreover, ${ }^{99 \mathrm{~m}} \mathrm{Tc}-\mathrm{NOET}$, a neutrally charged myocardial imaging agent, consists of a ${ }^{99 \mathrm{~m}} \mathrm{Tc}(\mathrm{V}) \mathrm{N}^{2+}$ core (Pasqualini et al., 1994). In addition, ${ }^{99 \mathrm{~m}} \mathrm{Tc}$-sestamibi (Cardiolite) has a +1 oxidation state and has octahedral coordination

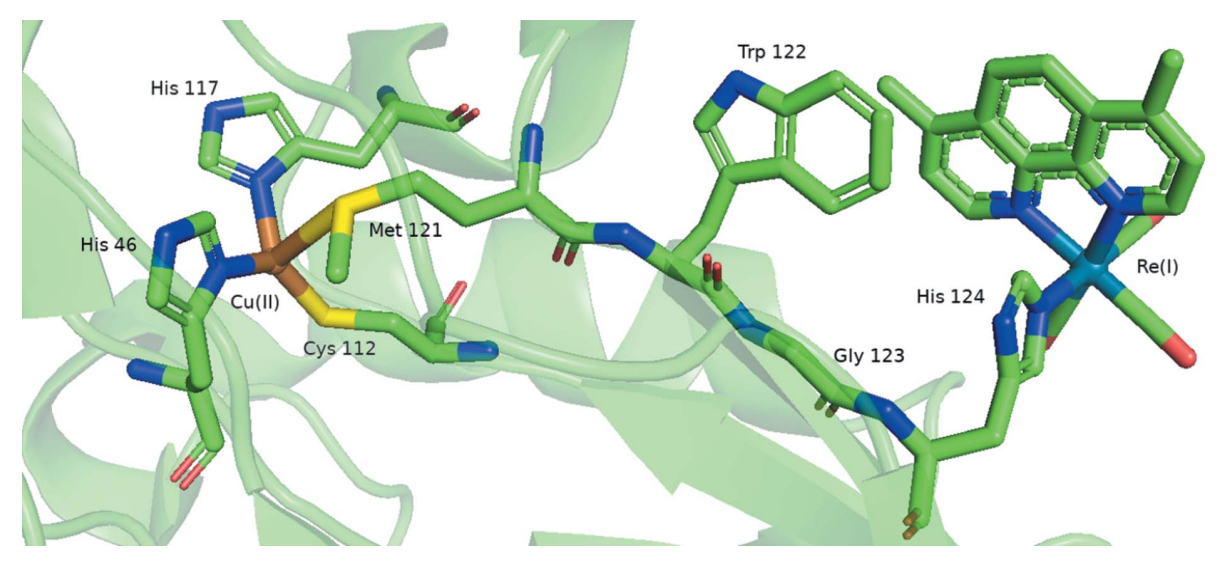

Figure 2

A coordination-site view of $2 \mathrm{i} 7 \mathrm{o}$ indicating both the coordination of a rhenium(I) tricarbonyl complex, $f a c$ - $\left[\mathrm{Re}^{\mathrm{I}}(\mathrm{CO})_{3}\right]$, via the histidine and the $\mathrm{Cu}{ }^{\mathrm{II}}$ to the azurin protein. This figure has been created with $P y M O L$. 


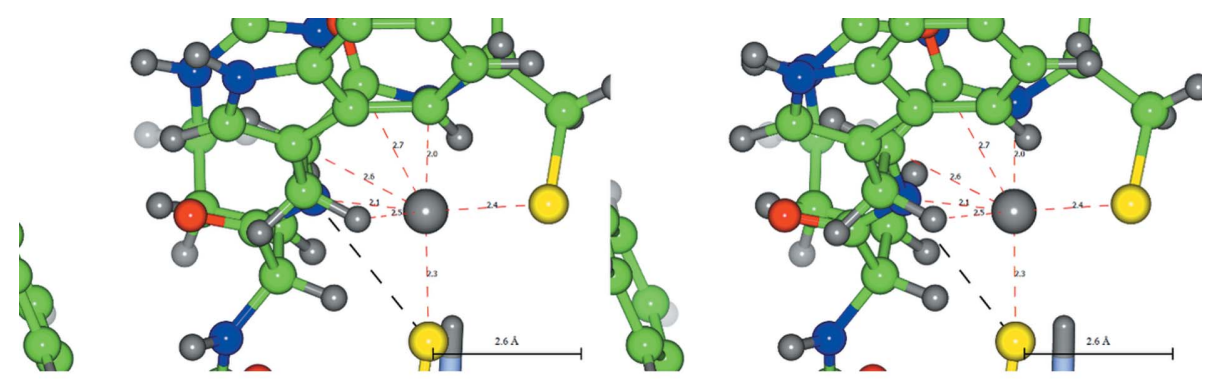

(a)

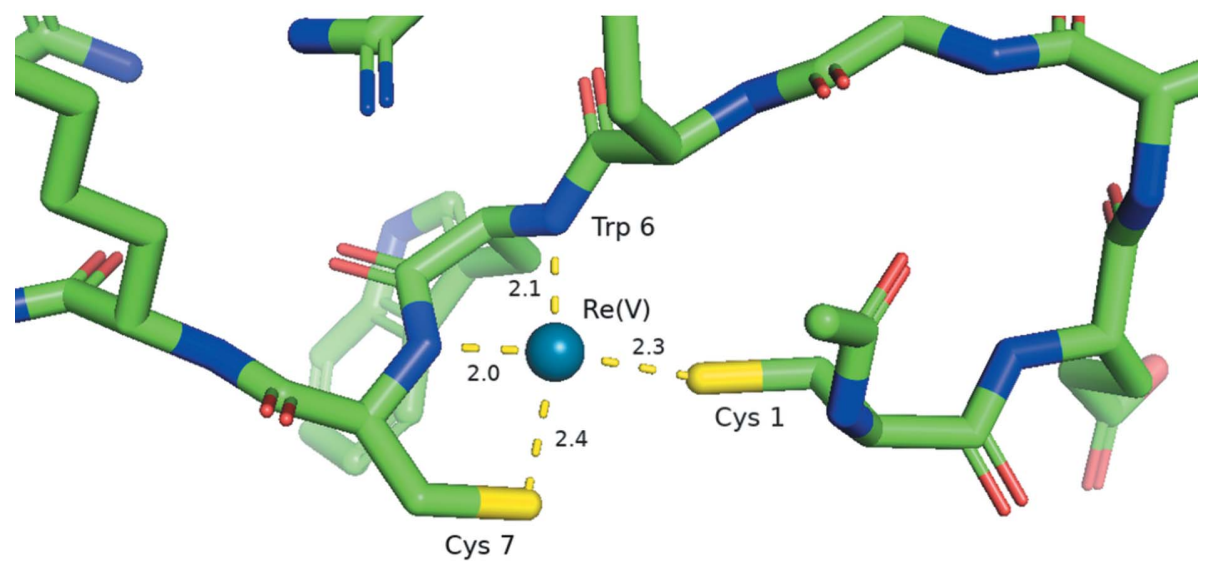

(b)

Figure 3

(a) A stereoview of 1b0q at the rhenium binding site. This part of the figure was created using CCP4mg (McNicholas et al., 2011). The distances shown are in $\AA$. (b) The binding site of 1b0q showing the cyclic coordination of $\operatorname{Re}(\mathrm{V})$ to the $\alpha$-MSH analog. This part of the figure was created using $P y M O L$. The distances shown are in $\AA$.

surrounded by isonitrile ligands. The water-soluble and readily synthesized $\mathrm{fac}_{-}\left[\mathrm{Tc}^{99 \mathrm{~m}}(\mathrm{CO})_{3}\right]^{+}$core from the IsoLink Kit (supplied by Mallinckrodt) similarly has a +1 oxidation state with octahedral coordination (Alberto et al., 1999, 2001; Schibli et al., 2000).

The oxidation states and transition-metal core play a key role in synthesis and solubility, as well as the general and biological chemistry (Liu, 2004; Alberto et al., 2020). We have therefore grouped and described the following medically applicable PDB structures according to the Re/Tc metal core which is found in these structures.

Caution: ${ }^{99} \mathrm{Tc}$ is a $\beta^{-}$emitter with a half life of $c a$ 210000 years, ${ }^{99 \mathrm{~m}} \mathrm{Tc}$ is a $\gamma$ emitter with a half life of $c a 6 \mathrm{~h}$, and ${ }^{186} \mathrm{Re}$ and ${ }^{188} \mathrm{Re}$ are $\beta^{-}$emitters with half lives of $c a 3.7 \mathrm{~d}$ and $17 \mathrm{~h}$, respectively. Thus, all experiments have to be performed in laboratories approved for working with low-level radioactive materials. Naturally occurring rhenium, ${ }_{75} \mathrm{Re}$, is $37.4 \%$ ${ }^{185} \mathrm{Re}$ (considered observably stable) and $62.6 \%{ }^{187} \mathrm{Re}$ (an unstable isotope but it has a very long half life of $c a 10^{10}$ years), it is therefore considered stable for standard laboratory use.

2.3.1. Radiopharmaceutical development utilizing rhenium oxo ( $\operatorname{Re} / \mathrm{Tc}^{\mathrm{V}} \mathrm{O}$ core) complex coordination to proteins. Radiopharmaceutical development utilizing rhenium-188 and technetium-99m metal coordination to proteins has been investigated by Giblin et al. (1998) utilizing the $\mathrm{Re} / \mathrm{Tc}^{\mathrm{V}}$ oxo core. The NMR study investigated the coordination of a
$\left[\mathrm{ReOCl}_{3}\left(\mathrm{Me}_{2} \mathrm{~S}\right)\left(\mathrm{OPPh}_{3}\right)\right]$ complex in solution. The authors' goal was to design ${ }^{188} \mathrm{Re}$ - or ${ }^{99 \mathrm{~m}}$ Tc-radiolabelled $\alpha$-melanocyte stimulating hormone $(\alpha-\mathrm{MSH})$ analogues in which metal coordination was an integral part of the molecule's structure (Fig. 3).

Both the Tc and Re oxo complexes in 1b0q (Giblin et al., 1998) were in the +5 oxidation state, which tends to prefer a square pyramidal coordination geometry. The cyclic Repeptide analogue, ReMSH, was synthesized by incorporating the $\mathrm{Re}^{\mathrm{V}} \mathrm{O}$ core into APOMSH via trans chelation from the $\left[\mathrm{ReOCl}_{3}\left(\mathrm{Me}_{2} \mathrm{~S}\right)\left(\mathrm{OPPh}_{3}\right)\right]$ organometallic complex. The $\alpha$ MSH analogues, cyclized through site-specific rhenium and technetium metal coordination, were structurally characterized and analysed for their ability to bind to $\alpha$-MSH receptors present on melanoma cells and in tumour-bearing mice. Crystal structure analysis of the Re-peptide complex showed that the disulfide bond of the original peptide was replaced by thiolate-metal-thiolate cyclization. When the metal binding site was redesigned, a second-generation Re-peptide complex (ReCCMSH) formed, which displayed a receptor binding affinity of $2.9 \mathrm{nM}, 25$-fold higher than the initial ReMSH analogue.

2.3.2. Protein coordination with perrhenate oxo cores involving molybdate substitution in molybdate-binding periplasmic protein. The protein coordination to an alternative rhenium core was explored by Aryal et al. (2012) (3axf) who presented a strategy to engineer proteins that may selectively 
recognize the perrhenate $\left(\mathrm{ReO}_{4}^{-}\right)$ion so as to develop a new method to label proteins. The $\mathrm{ReO}_{4}^{-}$anion is tetrahedral in shape and contains the rhenium atom in the +7 oxidation state with a $d^{0}$ configuration. It is similar in size and shape to perchlorate and the valence is isoelectronic to permanganate. It is also stable over a broad $\mathrm{pH}$ range (Eiroa-Lledo et al., 2020). The chemistry of the perrhenate ion is like that of the pertechnetate ion ${ }^{99 \mathrm{~m} / 99} \mathrm{TcO}_{4}^{-}$, which again makes it ideal for exploratory research without having to utilize the radioactive ${ }^{99 \mathrm{~m} / 99} \mathrm{Tc}$ radionuclide (Mazzi et al., 2007). The authors determined that the molybdate $\left(\mathrm{MoO}_{4}^{2-}\right)$ binding protein (ModA) from Escherichia coli can bind perrhenate with high affinity and were able to solve the crystal structure of ModA with a bound $\mathrm{ReO}_{4}^{-}$(3r26). The authors also synthesized a mutant protein containing a disulfide linkage, which exhibited increased affinity for the perrhenate (3axf). These protein structures both indicate that the $\mathrm{ReO}_{4}^{-}$ion occupies the $\mathrm{MoO}_{4}^{2-}$ binding site using the same amino-acid residues that

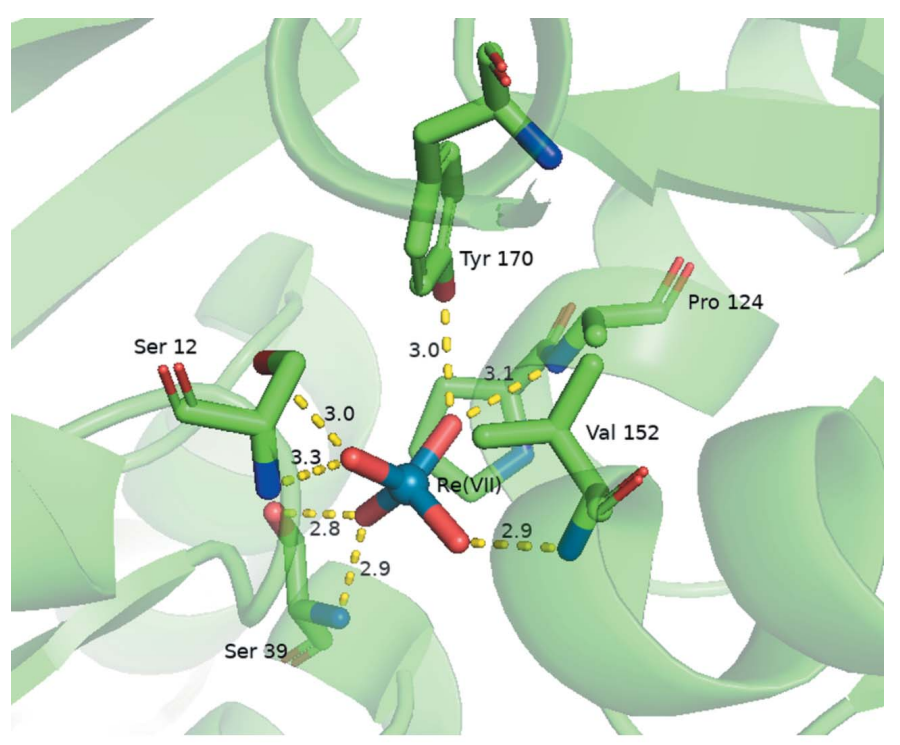

(a)

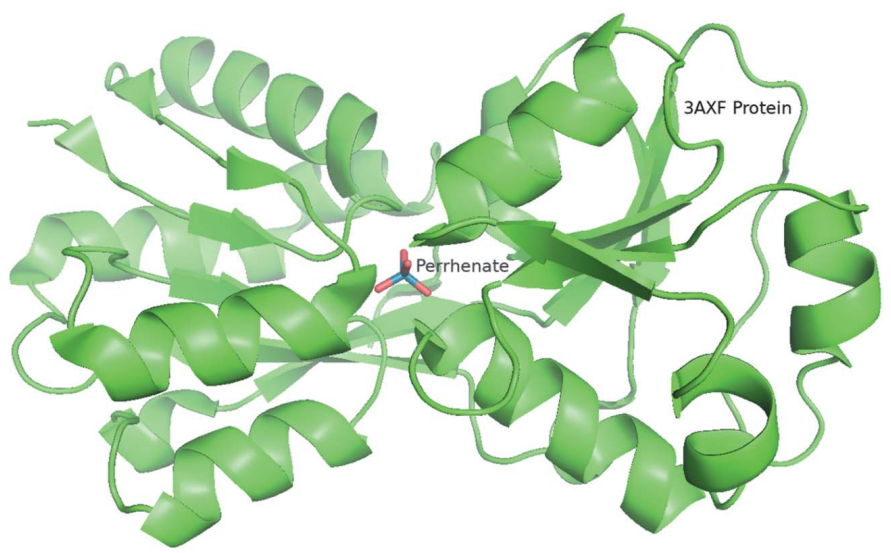

(b)

Figure 4

(a) A detailed view of the multiple weak interactions occurring between perrhenate and the protein residues of 3 axf. This was made using PyMOL. The distances shown are in $\AA$. (b) A distant view of the perrhenate situated overall in the protein. are involved in molybdate binding. The overall protein structure of the perrhenate-bound ModA is unchanged compared with that of the molybdate-bound form (see Fig. 4).

The affinity of most proteins for the radionuclides of rhenium and technetium is not known. The effect of the bifunctional chelator on the metal reactivity (Jacobs et al., 2021; Brink et al., 2014; Schutte et al., 2011, 2012) and the stabilities of the bidentate $[2+1] N, N^{\prime} ; N, O^{\prime} ; O, O^{\prime}$ or tridentate coordinated complexes under physiological conditions are still being explored (Schibli et al., 2000; Schibli \& Schubiger, 2002). These studies (3axf and 3r26) therefore make a valuable contribution to understanding molybdate protein interactions, particularly if it can be generalized so that more perrhenatebound proteins can selectively be stabilized with the presence of disulfide linkages. The authors indicate that the binding protein originates from a bacterium as the molybdate transporter in Homo sapiens has yet to be discovered. This could be applied for targeted delivery to an organ of concern, if other molybdate/perrhenate-labelled proteins could be identified. Secondly, the question arises if it would be possible to substitute the perrhenate oxo core $\left(\mathrm{ReO}_{4}^{-}\right)$with an alternative core, such as the $f a c-\left[\operatorname{Re}(\mathrm{CO})_{3}\right]^{+}$core that we have shown in our studies, summarized in Section 2.3.3, to be able to coordinate to multiple types of amino acids. This would increase the absorption and the clinical X-ray contrast.

2.3.3. Rhenium-protein coordination utilizing the fac$\left[\operatorname{Re}^{\prime}(\mathrm{CO})_{3}\right]$ core. The tricarbonyl cores of rhenium and technetium, $f a c-\left[M^{\mathrm{I}}(\mathrm{CO})_{3}\right](M=$ Re or Tc), are widely utilized due to their water solubility, as well as their relatively simple synthetic procedure which is conducted under mild conditions in aqueous solutions. The aqua complex $f a c-\left[M^{\mathrm{I}}(\mathrm{CO})_{3}\left(\mathrm{H}_{2} \mathrm{O}\right)_{3}\right]$ is coordinated by three tightly bound $\mathrm{CO}$ ligands and three labilizable water ligands. It is a highly attractive possibility for radiopharmaceutical design due to the high stability of the fac$\left[M(\mathrm{CO})_{3}\right]^{+}$core in water and the potential of exchanging the labile solvent ligands to allow coordination with many

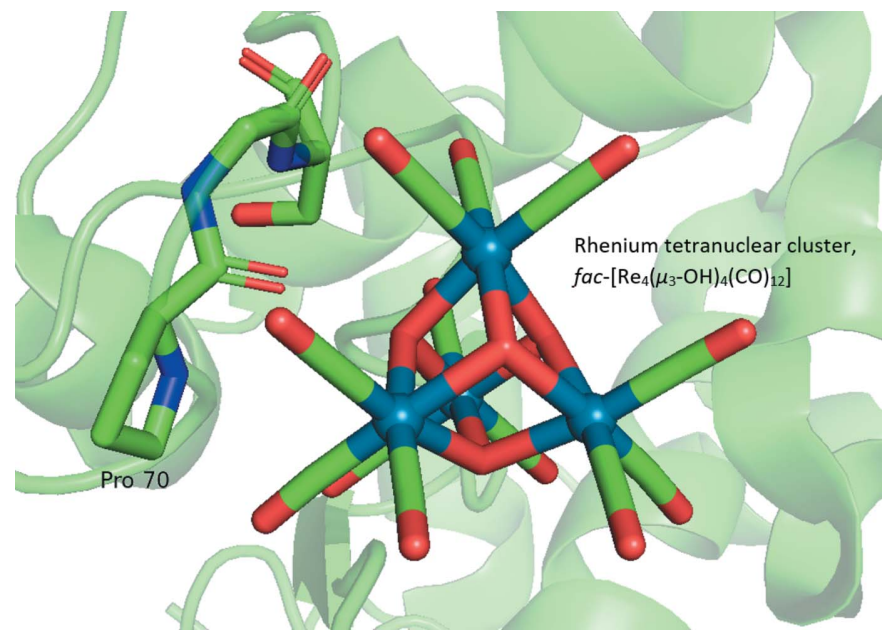

Figure 5

A PyMOL view of the rhenium tetranuclear cluster, $f a c-\left[\operatorname{Re}_{4}\left(\mu_{3^{-}}\right.\right.$ $\left.\mathrm{OH})_{4}(\mathrm{CO})_{12}\right]$, in the vicinity of Pro70 residue in the protein structure 6ro3. 


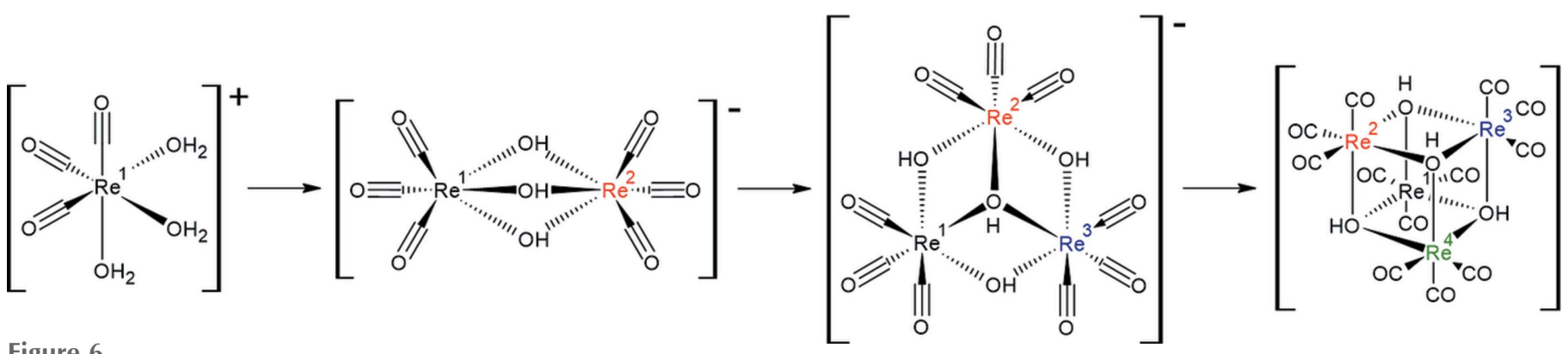

Figure 6

A schematic representation of the formation of the dinuclear, trinuclear and tetranuclear rhenium clusters starting from the $f a c$ - $\left[\mathrm{Re}(\mathrm{CO})_{3}\right]^{+} \mathrm{core}$. Each rhenium atom is coloured individually according to stepwise incorporation into the cluster; on its own at far left it is black, then second from left the added rhenium is red and so on for the third (blue) from left and the fourth one (green) at far right.

different types of ligands (Alberto et al., 1999, 2001; Schibli et al., 2000; Jacobs et al., 2021).

Within the wwPDB, 3rj7 (Can et al., 2012) describes rhenium bio-organometallic carbonic anhydrase inhibitors (CAI) with nanomolar affinities for specific CA subtypes. CAs are targets for cancer diagnosis and therapy because of hypoxia-induced overexpression of hCAIX and hCAXII ( $\mathrm{hCA}=$ human $\mathrm{CA})$ in several malignancies, including cancer (Lindskog, 1997; Supuran, 2008a,b; Bose \& Satyanarayana, 2017). In 3rj7, the study included both rhenium and technetium-99m arylsulfonamide, sulfamide, and sulfamate-based CAIs containing the $\left[(\mathrm{Cp}-\mathrm{R}) M(\mathrm{CO})_{3}\right]$ complex $(M=\mathrm{Re}$ or ${ }^{99 \mathrm{~m}} \mathrm{Tc} ; \mathrm{Cp}=$ cyclopentadienyl) (Can et al., 2012). All these complexes were in the +1 oxidation state and octahedral coordination. The $\left[(\mathrm{Cp}-\mathrm{R}) \operatorname{Re}(\mathrm{CO})_{3}\right]$ complex is found in the binding pocket of hCAII with no covalent bonds formed between the protein and the Re metal centre. However, the deprotonated nitrogen of the arylsulfonamide terminus of the $\left[(\mathrm{Cp}-\mathrm{R}) \operatorname{Re}(\mathrm{CO})_{3}\right]$ complex coordinates to the $\mathrm{Zn}$ atom in the active site, thus forming a protein-ligand bond. The $\left[(\mathrm{Cp}) \operatorname{Re}(\mathrm{CO})_{3}\right]$ complex has no further interactions with either the protein or water molecules (Can et al., 2012). However, there are hydrophobic interactions between the $\left[(\mathrm{Cp}) \operatorname{Re}(\mathrm{CO})_{3}\right]$ moiety and the hydrophobic parts of Phe131, Leu198 and Pro202 (RCSB NGL ligand viewer https://www.rcsb.org/docs/3d-viewers/ngl\#ligand-viewer-options) (Rose \& Hildebrand, 2015; Rose et al., 2017).

Other studies that have examined the coordination of $\mathrm{fac}$ $\left[\operatorname{Re}^{\mathrm{I}}(\mathrm{CO})_{3}\right]$ complexes to proteins, specifically to understand the protein-metal coordination for radiopharmaceutical development, have been described by Binkley et al. (2010) (3kam), Binkley et al. (2011), Zobi \& Spingler (2012) (3qng, 3qe8) and Santoro et al. (2012). The studies have shown that rhenium-protein coordination utilizing the $f a c-\left[\operatorname{Re}^{\mathrm{I}}(\mathrm{CO})_{3}\right]$ core has consistencies, such as the metal core showing binding preference to a histidine imidazole [Binkley et al., 2011; Zobi \& Spingler, 2012; Santoro et al., 2012; Takematsu et al., 2013 (structure $4 \mathrm{k} 9 \mathrm{j}$ studied for interest in electron tunnelling)]. The exception to the histidine imidazole sole preference was our study (Brink \& Helliwell, 2017), which employed two $\mathrm{X}$-ray wavelengths for rhenium resonant-scattering signal enhancement and enabled the finding of rhenium transitionmetal placements, even at low occupancy. With that approach, rhenium coordination was also observed in binding to aspartic acid, glutamic acid, arginine and leucine residues (5nbj; Brink \& Helliwell, 2017). The kinetic formation of tetranuclear rhenium clusters appropriate for theranostic applications, albeit in the crystal and rather slow (up to two years), has also been observed with the $f a c-\left[\operatorname{Re}^{\mathrm{I}}(\mathrm{CO})_{3}\right]$ core (6ro3, 6ro5; Brink \& Helliwell, 2019a) (see Fig. 5).

Fig. 6 summarizes the complete kinetic stepwise formation that the rhenium complexes can undergo, and where the mono- and tetranuclear complexes $\left\{\mathrm{fac}-\left[\mathrm{Re}(\mathrm{CO})_{3}\right]^{+}\right.$and $\mathrm{fac}$ $\left.\left[\operatorname{Re}_{4}\left(\mu_{3}-\mathrm{OH}\right)_{4}(\mathrm{CO})_{12}\right]\right\}$ were observed in the protein-rhenium crystal structures studied. We deem this expanded group of rhenium complexes seen bound to a protein as a breakthrough in the whole field, particularly as it is synthetically possible to substitute one rhenium atom with either technetium-99m or technetium-99 to form a mixed rhenium and technetium version where more than one metal centre is present and with possible further theranostic applications (Mokolokolo et al., 2018; Frei et al., 2018).

Of additional medical interest is the recent report of the synthesis and biophysical evaluation of a series of fac$\left[\operatorname{Re}^{\mathrm{I}}(\mathrm{CO})_{3}(\text { bipy })\right]^{+}$(bipy $=2,2$ bipyridine ligand) complexes as inhibitors of the SARS-CoV-2 main protease $3 \mathrm{CL}^{\text {pro }}$ (3-chymotrypsin-like protease) (Karges et al., 2021). Massspectrometry experiments verified the covalent binding of a single $\left[\operatorname{Re}^{\mathrm{I}}(\mathrm{CO})_{3}\right]$ complex to the $3 \mathrm{CL}^{\text {pro }}$ preferentially via the Cys145 amino acid. The authors suggest that rhenium(I) tricarbonyl complexes can serve as a starting scaffold for the development of potent selective SARS-CoV-2 inhibitors.

\section{Forward look: what is the best way for this field to progress?}

We have stated the need to identify any possible trends that may be occurring in this field as they could provide key information on whether there is any binding preference occurring between the group 7 transition-metal series and proteins. The themes of other research labs have been described and particularly the reasons as to why rhenium was chosen. It is also important to note the variety of proteins used, the wide range of organometallic complexes and the crystallization conditions (Table 1). 
In our research, we have firstly identified that there are more amino-acid types binding to rhenium organometallics than previously seen. Secondly, via our most recent research, we have expanded the available repertoire of rhenium compounds to include multi-metal-centre complexes (Brink \& Helliwell, 2017, 2019a). Both these advances have the potential to increase the absorption of the organometallic complex at the organ being imaged. These are promising steps forward for reducing the overall medical-imaging radiation exposure needed, as well as the potential for creating a dual drug, one containing both imaging and therapeutic applications via the inclusion of $\mathrm{Re}$ and $\mathrm{Tc}$ metal centres. The toxicity evaluation of any new compound is a major defining step as to whether a new compound has a commercial future or not and requires the take up of the frontline research into any pharmaceutical company's research and development program (PhRMA, 2015; Eckstein, 2005; Tonkens, 2005; Torjesen, 2015).

The challenge for the chemist is how to localize the organometallic binding to the cancerous cells but not the normal tissue. Specific area injection is an obvious answer to this challenge, such as the use of heterogeneous ${ }^{188} \mathrm{Re}$-colloids for brachytherapy, which can be physically inserted at a site (Lepareur et al., 2019). Another is the continued development of site-specific complexes (Liu, 2004). Agents that bind to a specific site in the biological organ with high concentration cause minimal damage to the surrounding tissue. This review clearly highlights an unusual commonality that supports the ideal of the latter suggestion. Of the 27 PDB entries containing rhenium listed in Table 1,74\% of these (i.e. 20 structures) show direct coordination of the metal to a histidine moiety via the imidazole group. This is a marked preference for one particular amino-acid residue, particularly when considering that crystallization conditions were markedly variable and involved various organometallic cores and oxidation states. Furthermore, 11 different proteins were analysed containing basically a full range of amino-acid residue types on their protein surfaces.

So, how might this whole field progress? Fundamentally there are some significant technical obstacles from a crystallographic aspect that must be addressed in future research.

Firstly, data have been extracted from the PDB over a broad time period spanning 20 years or more. Significant scientific and technology progress has occurred during this period, including in crystallization techniques, X-ray synchrotron/lasers/home sources, detectors, software developments, IUCr publication and validation requirements, CSD/ wwPDB data submission and validation requirements etc. We also wish to emphasize the need of FAIR data principles (where FAIR data is findable, accessible, interoperable and reusable) in the field of macromolecular and chemical crystallography. The purpose of our review is not to criticize the authors of past articles we have referred to who did not have the tools available today. But we ask the question how can published data and tools currently made available to macromolecular crystallographers be interoperably utilized by scientists in disciplines other than the original purpose (Helliwell, 2019)? We have found that not all of our surveyed database deposits are 'reusable' since they do not contain the structure factors, a point we return to below.

Accurately examining multiple weak interactions should be extractable in either macromolecular or chemical crystallography and is crucial for drug development. A valuable tool provided by the CSD is CSD-CrossMiner. Care should be taken when viewing possible trends in protein-metal interactions, particularly when searching for $d$-block transition metals as carried out for this study. In such an organometallics review, we recommend a combined analysis is carried out via the stepwise-analyse-by-hand method, supported by the available search engines developed by the chemical and/or macromolecular crystallographic community to avoid missing any key information. Another future development that would be most useful is the availability of constructing space-volume calculations from small molecules and then being able to search for identical 'space-volume pockets' on proteins in their structures downloaded from the PDB. Thus, both electronic and steric factors could be examined either individually or collectively, a factor utilized in homogeneous catalysis research with calculations such as the Tolman cone angle (Tolman, 1977; Bilbrey et al., 2013).

Thirdly, we see the need to extend our research, and the studies by others, to where the whole crystallography procedure is undertaken ideally at mammalian body temperature $\left(37^{\circ} \mathrm{C}\right)$. This is quite challenging because the co-crystallization of the organometallic of interest with a protein should also be carried out at $37^{\circ} \mathrm{C}$, not only the X-ray diffraction data collection. The crystallization conditions at room temperature ( $\sim 20$ to $25^{\circ} \mathrm{C}$ ) may not be the same at $37^{\circ} \mathrm{C}$. Organometallic reaction-rate constants generally increase by a factor of two or three for each $10^{\circ} \mathrm{C}$ rise in temperature (Moore \& Pearson, 1981). Such studies will assess whether the weaker occupancy binding sites would have increased metal occupation at body temperature or would migration to the dominant binding species (i.e. histidine) become more prominent? Also, could structure studies of proteins at variable temperatures increase our understanding of dynamic movements by examining the flexibility of side chains, or by the loss of water molecules (Helliwell, 2021; Tilton et al., 1992, Sanchez et al., 2019)? A recent review describes the practical aspects of preparing, acquiring and analysing X-ray crystallography data at room temperature, and sheds light on preconceived impracticalities that tend to deter most crystallographers from conducting routine room-temperature data collection at synchrotron sources (Fischer, 2021).

Fourthly, a fundamental difficulty of evaluating the precision of quite a number of PDB entries is the absence of their associated structure-factor files (such as in $1 \mathrm{k} 4 \mathrm{j}, 1 \mathrm{i} 53,1 \mathrm{jzi}, 1 \mathrm{r} 1 \mathrm{c}$ and $2 \mathrm{fnw}$ ). Thus, the import of a particular PDB entry into Coot (Emsley \& Cowtan, 2004) does not yield the difference electron-density map in such cases. To examine the difference electron-density map is vital for seeing features that are not the focus of the authors' model (or original purpose) and specifically to check if there are any signs of structural disorder around the rhenium sites or possibly more weakly occupied metal sites. Specifically of relevance to this review is 
the question: could these disorders be eliminated and the rhenium compound harnessed to better advantage for a radiopharmaceutical biomedical application? If the structure factors are available to re-refine the model, this is not necessarily straightforward if the ligand restraints file is not available. The lack of interoperability of the PDB and the CSD in such a situation can be a considerable obstacle (Brink \& Helliwell, 2019b). Additional differences are observed between the authors indicating formal protein-metal bonding and protein-ligand interactions versus the RCSB NGL ligand viewer (Rose \& Hildebrand, 2015; Rose et al., 2017), which lists the weak interactions. The RCSB PDB clearly defines the criteria for the interaction types and the calculation parameters used. To gain greater clarity between possible discrepancies of this kind, it would be best to analyse the precision of each bond distance, factoring in the resolution of each PDB entry, diffraction data completeness etc., to accurately determine which is a weak interaction and which is a formal bond.

And finally, it is important to note that absolute configuration is a key aspect affecting the chemistry of organometallic or inorganic compounds and therefore must be correctly illustrated or described. Many database entries interchangeably utilize SMILES or InChI notation when constructing 2D diagrams (or ligand CIFs for protein refinement). However, organometallic complexes are problematic to describe because their bonding scheme cannot fully be explained by valence-bond theory (David et al., 2020). It is sometimes difficult (utilizing the notation) to clearly establish which atoms of the ligands are bound to the metal and to decide which bond-order scheme suits the specific organometallic compound the best (Quirós et al., 2018). This often leads to ambiguity in representation when algorithms or automatic machine drawing tools are utilized (Heller et al., 2015). It is therefore strongly recommended to always refer to the original publication and the PDB entry/CIF to view the correct organometallic configuration.

We hope that this topical review survey and descriptions of possible improvements to the methods will stimulate this important field for further, even enhanced, medical application.

\section{Acknowledgements}

Opinions, findings, conclusions or recommendations expressed in this material are those of the authors and do not necessarily reflect the views of the South African National Research Foundation (SA NRF). JRH is very grateful to the University of Manchester for support. We thank the Diamond Light Source for synchrotron radiation within the University of Manchester Block Allocation Group coordinated by Dr Colin Levy, who we also thank.

\section{Funding information}

$\mathrm{AB}$ and FJFJ wish to thank the SA NRF, the Research Fund of the University of the Free State and SASOL for financial support.

\section{References}

Abe, S., Hirata, K., Ueno, T., Morino, K., Shimizu, N., Yamamoto, M., Takata, M., Yashima, E. \& Watanabe, Y. (2009). J. Am. Chem. Soc. 131, 6958-6960.

Alberto, R., Braband, H. \& Nadeem, Q. (2020). Chimia, 74, 953-959.

Alberto, R., Ortner, K., Wheatley, N., Schibli, R. \& Schubiger, A. P. (2001). J. Am. Chem. Soc. 123, 3135-3136.

Alberto, R., Schibli, R., Schubiger, A. P., Abram, U., Pietzsch, H. \& Johannsen, B. (1999). J. Am. Chem. Soc. 121, 6076-6077.

Aliyan, A., Paul, T. J., Jiang, B., Pennington, C., Sharma, G., Prabhakar, R. \& Martí, A. A. (2017). Chem, 3, 898-912.

Aryal, B. P., Brugarolas, P. \& He, C. (2012). J. Biol. Inorg. Chem. 17, 97-106.

Bastard, K., Isabet, T., Stura, E. A., Legrand, P. \& Zaparucha, A. (2018). Sci. Rep. 8, 16587-16598.

Bilbrey, J. A., Kazez, A. H., Locklin, J. \& Allen, W. D. (2013). J. Comput. Chem. 34, 1189-1197.

Binkley, S. L., Leeper, T. C., Rowlett, R. S., Herrick, R. S. \& Ziegler, C. J. (2011). Metallomics, 3, 909-916.

Binkley, S. L., Ziegler, C. J., Herrick, R. S. \& Rowlett, R. S. (2010). Chem. Commun. 46, 1203-1205.

Blanco-Rodríguez, A. M., Busby, M., Grǎdinaru, C., Crane, B. R., Di Bilio, A. J., Matousek, P., Towrie, M., Leigh, B. S., Richards, J. H., Vlček, A. \& Gray, G. B. (2006). J. Am. Chem. Soc. 128, 4365-4370.

Blanco-Rodríguez, A. M., Busby, M., Ronayne, K., Towrie, M., Grădinaru, C., Sudhamsu, J., Sýkora, J., Hof, M., Záliš, S., Di Bilio, A. J., Crane, B. R., Gray, H. B. \& Vlček, A. (2009). J. Am. Chem. Soc. 131, 11788-11800.

Bose, H. \& Satyanarayana, T. (2017). Front. Microbiol. 8, 1615.

Boswell, C. A. \& Brechbiel, M. W. (2007). Nucl. Med. Biol. 34, 757-778.

Brink, A. \& Helliwell, J. R. (2017). IUCrJ, 4, 283-290.

Brink, A. \& Helliwell, J. R. (2019a). IUCrJ, 6, 695-702.

Brink, A. \& Helliwell, J. R. (2019b). IUCrJ, 6, 788-793.

Brink, A., Visser, H. G. \& Roodt, A. (2014). Inorg. Chem. 53, 12480-12488.

Can, D., Spingler, B., Schmutz, P., Mendes, F., Raposinho, P., Fernandes, C., Carta, F., Innocenti, A., Santos, I., Supuran, C. T. \& Alberto, R. (2012). Angew. Chem. Int. Ed. 51, 3354-3357.

Carreño, A., Gacitúa, M., Solis-Céspedes, E., Páez-Hernández, D., Swords, W. B., Meyer, G. J., Preite, M. D., Chávez, I., Vega, A. \& Fuentes, J. A. (2021). Front. Chem. 9, 647816.

Chayen, N. E., Helliwell, J. R. \& Snell, E. H. (2010). Macromolecular Crystallization and Crystal Perfection, IUCr Monographs on Crystallography, No. 24. Oxford University Press.

Chi, D. Y. \& Katzenellenbogen, J. A. (1993). J. Am. Chem. Soc. 115, 7051-7052.

Chi, D. Y., O'Neil, J. P., Anderson, C. J., Welch, M. J. \& Katzenellenbogen, J. A. (1994). J. Med. Chem. 37, 928-937.

Chung, T. D. Y., Terry, D. B. \& Smith, L. H. (2015). In Assay Guidance Manual, edited by S. Markossian, A. Grossman, K. Brimacombe et al. Bethesda: Eli Lilly \& Company and the National Center for Advancing Translational Sciences.

Ciccone, L., Policar, C., Stura, E. A. \& Shepard, W. (2016). J. Struct. Biol. 195, 353-364.

Crane, B. R., Di Bilio, A. J., Winkler, J. R. \& Gray, H. B. (2001). J. Am. Chem. Soc. 123, 11623-11631.

Daubit, I. M., Sullivan, M. P., John, M., Goldstone, D. C., Hartinger, C. G. \& Metzler-Nolte, N. (2020). Inorg. Chem. 59, 17191-17199.

David, L., Thakkar, A., Mercado, R. \& Engkvist, O. (2020). J. Cheminform, 12, 56.

DeLano, W. L. (2002). PyMOL. http://www.pymol.org.

Di Bilio, A. J., Crane, B. R., Wehbi, W. A., Kiser, C. N., Abu-Omar, M. M., Carlos, R. M., Richards, J. H., Winkler, J. R. \& Gray, H. B. (2001). J. Am. Chem. Soc. 123, 3181-3182.

Dilworth, J. R. \& Parrott, S. J. (1998). Chem. Soc. Rev. 27, 43. 
DiZio, J. P., Anderson, C. J., Davison, A., Ehrhardt, G. J., Carlson, K. E., Welch, M. J. \& Katzenellenbogen, J. A. (1992). J. Nucl. Med. 33, $558-569$.

Dodds, W. J. \& Powell, M. R. (1968). Radiology, 91, 27-31.

Eckstein, J. (2005). ISOA/ARF Drug Development Tutorial, https:// www.alzforum.org/sites/default/files/legacy/drg/tut/ISOATutorial.pdf.

Eichinger, A., Haneburger, I., Koller, C., Jung, K. \& Skerra, A. (2011). Protein Sci. 20, 656-669.

Eiroa-Lledo, C., Lecrivain, L., Parker, T. G., Wall, D. E. \& Wall, N. A. (2020). Radiochim. Acta, 108, 443-449.

Emsley, P. \& Cowtan, K. (2004). Acta Cryst. D60, 2126-2132.

Erlanson, D. A. (2012). Fragment-Based Drug Discovery and X-ray Crystallography, edited by T. G. Davies \& M. Hyvönen, pp. 1-32. Heidelberg: Springer.

Firestone, R. B., Shirley, V. S., Baglin, S. B., Chu, S. Y. F. \& Zipkin, J. (1996). Table of Isotopes, 8th ed. New York: Wiley.

Fischer, M. (2021). Q. Rev. Biophys. 54, e1, 1-15.

Frei, A., Mokolokolo, P. P., Bolliger, R., Braband, H., Tsosane, M. S., Brink, A., Roodt, A. \& Alberto, R. (2018). Chem. Eur. J. 24, 10397-10402.

Gerson, M. C., Deutsch, E. A., Nishiyama, H., Libson, K. G., Adolph, R. J., Grossman, L. W., Sodd, V. J., Fortman, J. L., Vanderhagen, C., Williams, C. C. \& Saenger, E. L. (1983). J. Nucl. Med. 8, 371-374.

Giblin, M. F., Wang, N., Hoffman, T. J., Jurisson, S. S. \& Quinn, T. P. (1998). Proc. Natl Acad. Sci. USA, 95, 12814-12818.

Goffin, K. E., Joniau, S., Tenke, P., Slawin, K., Klein, E. A., Stambler, N., Strack, T., Babich, J., Armor, T. \& Wong, V. (2017). J. Nucl. Med. 58, 1408-1413.

Heller, S. R., McNaught, A., Pletnev, I., Stein, S. \& Tchekhovskoi, D. (2015). J. Cheminform, 7, 23.

Helliwell, J. R. (2019). Struct. Dyn. 6, 054306-1.

Helliwell, J. R. (2021). Acta Cryst. A77, 173-185.

Herman, L. W., Sharma, V., Kronauge, J. F., Barbarics, E., Herman, L. A. \& Piwnica-Worms, D. (1995). J. Med. Chem. 38, 2955-2963.

Holman, B. L., Jones, A. G., Lister-James, J., Davison, A., Abrams, M. J., Kirshenbaum, J. M., Tumeh, S. S. \& English, R. J. (1984). J. Nucl. Med. 25, 1350-1355.

Jacobs, F. J. F., Venter, G. J. S., Fourie, E., Kroon, R. E. \& Brink, A. (2021). RSC Adv. 11, 24443-24455.

Jones, G., Willett, P., Glen, R. C., Leach, A. R. \& Taylor, R. (1997). J. Mol. Biol. 267, 727-748.

Joseph-McCarthy, D., Campbell, A. J., Kern, G. \& Moustakas, D. (2014). J. Chem. Inf. Model. 54, 693-704.

Jurisson, S., Berning, D., Jia, W. \& Ma, D. (1993). Chem. Rev. 93, 1137-1156.

Karges, J., Kalaj, M., Gembicky, M. \& Cohen, S. M. (2021). Angew. Chem. Int. Ed. 60, 10716-10723.

Kelly, J. D., Forster, A. M., Higley, B., Archer, C. M., Booker, F. S., Canning, L. R., Chiu, K. W., Edwards, B., Gill, H. K., McPartlin, M., Nagle, K. R., Latham, I. A., Pickett, R. D., Storey, A. E. \& Webbon, P. M. (1993). J. Nucl. Med. 34, 222-227.

Kluba, C. A. \& Mindt, T. L. (2013). Molecules, 18, 3206-3226.

Lam, M. G. E. H., Bosma, T. B., van Rijk, P. P. \& Zonnenberg, B. A. (2009). Eur. J. Nucl. Med. Mol. Imaging, 36, 1425-1433.

Lambert, B., Bacher, K., De Keukeleire, K., Smeets, P., Colle, I., Jeong, J. M., Thierens, H., Troisi, R., De Vos, F. \& Van de Wiele, C. (2005). J. Nucl. Med. 46, 1326-1332.

Lepareur, N., Lacoeuille, F., Bouvry, C., Hindré, F., Garcion, E., Chérel, M., Noiret, N., Garin, E. \& Russ Knapp Jr., F. F. (2019). Front. Med. 6, 132-151.

Liepe, K., Runge, R. \& Kotzerke, J. (2005). J. Cancer Res. Clin. Oncol. 131, 60-66.

Lin, X., Ruan, Q., Lin, L., Zhang, X., Duan, X., Teng, Y. \& Zhang, J. (2018). J. Radioanal. Nucl. Chem. 317, 1463-1468.

Lindskog, S. (1997). Pharmacol. Ther. 74, 1-20.

Liu, S. (2004). Chem. Soc. Rev. 33, 445-461.

Loreto, D., Ferraro, G. \& Merlino, A. (2021). Int. J. Mol. Sci. 22, 1496-1512.
Maltarollo, V. G., Gertrudes, J. C., Oliveira, P. R. \& Honorio, K. M. (2015). Expert Opin. Drug Metab. Toxicol. 11, 259-271.

Mazzi, U., Schibli, R., Pietzsch, H. J., Künstler, J.-U. \& Spies, H. (2007). Technetium-99m Pharmaceuticals: Preparation and Quality Control in Nuclear Medicine, edited by I. Zolle, pp. 7-58. Heidelberg: Springer.

McNicholas, S., Potterton, E., Wilson, K. S. \& Noble, M. E. M. (2011). Acta Cryst. D67, 386-394.

Messori, L. \& Merlino, A. (2016). Coord. Chem. Rev. 315, 67-89.

Miller, J. E., Grdinaru, C., Crane, B. R., Di Bilio, A. J., Wehbi, W. A., Un, S., Winkler, J. R. \& Gray, H. B. (2003). J. Am. Chem. Soc. 125, 14220-14221.

Mokolokolo, P. P., Frei, A., Tsosane, M. S., Kama, D. V., SchutteSmith, M., Brink, A., Visser, H. G., Meola, G., Alberto, R. \& Roodt, A. (2018). Inorg. Chim. Acta, 471, 249-256.

Monteiro, L. O. F., Fernandes, R. S., Castro, L. C., Cardoso, V. N., Oliveira, M. C., Townsend, D. M., Ferretti, A., Rubello, D., Leite, E. A. \& de Barros, A. L. B. (2017). Biomed. Pharmacother. 89, 146-151.

Moore, J. W. \& Pearson, R. G. (1981). Kinetics and Mechanism, 3rd ed. New York: Wiley.

Murray, C. W., Verdonk, M. L. \& Rees, D. C. (2012). Trends Pharmacol. Sci. 33, 224-232.

Mursula, A. M., van Aalten, D. M. F., Hiltunen, J. K. \& Wierenga, R. K. (2001). J. Mol. Biol. 309, 845-853.

Nogrady, T. \& Weaver, D. F. (2005). Medicinal Chemistry: A Molecular and Biochemical Approach, 3rd ed. Oxford University Press.

Oliveira, B. L., Moreira, I. S., Fernandes, P. A., Ramos, M. J., Santos, I. \& Correia, J. D. G. (2013). J. Mol. Graphics Modell. 45, 13-25.

Pasqualini, R., Duatti, A., Bellande, E., Comazzi, V., Brucato, V., Hoffschir, D., Fagret, D. \& Comet, M. (1994). J. Nucl. Med. 35, 334-341.

PhRMA (2015). Biopharmaceutical Research \& Development: The Process Behind New Medicines, http://phrma-docs.phrma.org/sites/ default/files/pdf/rd_brochure_022307.pdf.

Piwnica-Worms, D., Rao, V. V., Kronauge, J. F. \& Croop, J. M. (1995). Biochemistry, 34, 12210-12220.

Quirós, M., Gražulis, S., Girdzijauskaitè, S., Merkys, A. \& Vaitkus, A. (2018). J. Cheminform, 10, 23.

Rose, A. S. \& Hildebrand, P. W. (2015). Nucleic Acids Res. 43, W576-W579.

Rose, P. W., Prlić, A., Altunkaya, A., Bi, C., Bradley, A. R., Christie, C. H., Costanzo, L., Duarte, J. M., Dutta, S., Feng, Z., Green, R. K., Goodsell, D. S., Hudson, B., Kalro, T., Lowe, R., Peisach, E., Randle, C., Rose, A. S., Shao, C., Tao, Y. P., Valasatava, Y., Voigt, M., Westbrook, J. D., Woo, J., Yang, H., Young, J. Y., Zardecki, C., Berman, H. M. \& Burley, S. K. (2017). Nucleic Acids Res. 45, D271-D281.

Ruiz-Garcia, A., Bermejo, M., Moss, A. \& Casabo, V. G. (2008). J. Pharm. Sci. 97, 654-690.

Sanchez, J. C., Carrillo, M., Pandey, S., Noda, M., Aldama, L., Feliz, D., Claesson, E., Wahlgren, W. Y., Tracy, G., Duong, P., Nugent, A. C., Field, A., Šrajer, V., Kupitz, C., Iwata, S., Nango, E., Tanaka, R., Tanaka, T., Fangjia, L., Tono, K., Owada, S., Westenhoff, S., Schmidt, M. \& Stojković, E. A. (2019). Struct. Dyn. 6, 054701.

Santoro, G., Blacque, O. \& Zobi, F. (2012). Metallomics, 4, 253-259.

Schibli, R., La Bella, R., Alberto, R., Garcia-Garayoa, E., Ortner, K., Abram, U. \& Schubiger, P. A. (2000). Bioconjugate Chem. 11, 345-351.

Schibli, R. \& Schubiger, A. P. (2002). Eur. J. Nucl. Med. Mol. Imaging, 29, 1529-1542.

Schutte, M., Kemp, G., Visser, H. G. \& Roodt, A. (2011). Inorg. Chem. 50, 12486-12498.

Schutte, M., Roodt, A. \& Visser, H. G. (2012). Inorg. Chem. 51, 11996-12006.

Shih, C., Museth, K. A., Abrahamsson, M., Blanco-Rodriguez, A. M., Di Bilio, A. J., Sudhamsu, J., Crane, B. R., Ronayne, K. L., Towrie, 
M., Vlček, A. J., Richards, J. H., Winkler, J. R. \& Gray, H. B. (2008). Science, 320, 1760-1762.

Stuchebrukhov, A. A. (2010). Laser Phys. 20, 125-138.

Supuran, C. T. (2008a). Nat. Rev. Drug Discov. 7, 168-181.

Supuran, C. T. (2008b). Curr. Pharm. Des. 14, 603-614.

Takematsu, K., Williamson, H., Blanco-Rodríguez, A. M., Sokolová, L., Nikolovski, P., Kaiser, J. T., Towrie, M., Clark, I. P., Vlček, A., Winkler, J. R. \& Gray, H. B. (2013). J. Am. Chem. Soc. 135, $15515-15525$.

Takematsu, K., Williamson, H. R., Nikolovski, P., Kaiser, J. T., Sheng, Y., Pospíśil, P., Towrie, M., Heyda, J., Hollas, D., Záliš, S., Gray, H. B., Vlček, A. \& Winkler, J. R. (2019). ACS Cent. Sci. 5, 192-200.

Tanley, S. W. M., Starkey, L.-V., Lamplough, L., Kaenket, S. \& Helliwell, J. R. (2014). Acta Cryst. F70, 1132-1134.

Tezcan, F. A., Crane, B. R., Winkler, J. R. \& Gray, H. B. (2001). Proc. Natl Acad. Sci. USA, 98, 5002-5006.
Groom, C. R., Bruno, I. J., Lightfoot, M. P. \& Ward, S. C. (2016). Acta Cryst. B72, 171-179.

Tilton, R. F. Jr, Dewan, J. C. \& Petsko, G. A. (1992). Biochemistry, 31, 2469-2481.

Tolman, C. A. (1977). Chem. Rev. 77, 313-348.

Tonkens, R. (2005). Phys. Exec. 31, 48-52.

Torjesen, I. (2015). The Pharmaceutical Journal, https://doi:10.1211/ PJ2015.20068196.

Tuntland, T., Ethell, B., Kosaka, T., Blasco, F., Zang, R., Jain, M., Gould, T. \& Hoffmaster, K. (2014). Front. Pharmacol. 5, 1-16.

Wang, M., Moynié, L., Harrison, P., Kelly, V., Piper, A., Naismith, J. H. \& Campopiano, D. J. (2017). Nat. Chem. Biol. 13, 660-667.

Watson, W. T., Minogue, T. D., Val, D. L., von Bodman, S. B. \& Churchill, M. E. A. (2002). Mol. Cell, 9, 685-694.

Zobi, F. \& Spingler, B. (2012). Inorg. Chem. 51, 1210-1212. 